\title{
The Ulysses Catalog of Solar Hard X-Ray Flares
}

\author{
C. Tranquille $\cdot$ K. Hurley $\cdot$ H.S. Hudson
}

Received: 16 March 2007 / Accepted: 27 May 2009 / Published online: 19 June 2009

(C) The Author(s) 2009. This article is published with open access at Springerlink.com

\begin{abstract}
Ulysses was launched in October 1990, and its Solar X-ray/Cosmic Gamma-Ray Burst Experiment (GRB) has provided more than 13 years of uninterrupted observations of solar X-ray flare activity. Due to the large variation of the relative solar latitude and longitude of the spacecraft orbit with respect to the Earth, the perspective of the GRB instrument often differed significantly from that of X-ray instruments on Earth-orbiting satellites. During extended periods the GRB experiment made direct observations of flares on the hidden face of the Sun, providing a unique record of events not visible to other instruments. The small detector area of GRB and its optimization for very high counting rates minimized the effects of pulse pile-up. We interpret the spectra, time histories, and occurrence distribution patterns of GRB data in terms of "thermal feed-through", the confusion of thermal soft X-rays and non-thermal hard X-rays. This effect is a systematic problem for scintillation-counter spectrometers observing the solar hard X-ray spectrum. This paper provides a definitive catalog of the Ulysses X-ray flare observations and discusses various features of this unique database. For the equivalent GOES range X2 - X25, we find a power-law fit for the (differential) occurrence frequency at $>25 \mathrm{keV}$ with slope $-1.61 \pm 0.04$, with no evidence for a downturn at the highest event magnitudes (for the relatively small sample of such events available in this study). If the nine most intense events are excluded because of concerns about the effects of pulse pile-up, the slope steepens to $-1.75 \pm 0.08$.
\end{abstract}

\section{Introduction}

Solar hard X-rays result from the interaction of energetic electrons with ions present in the ambient solar atmosphere (e.g., Dennis, 1985; Hudson and Ryan, 1995). Electrons, accelerated to energies above $\approx 20 \mathrm{keV}$ by processes not yet properly understood, emit continuum

C. Tranquille (凶)

Research and Scientific Support Department, ESA/ESTEC, Postbus 299, 2200 AG, Noordwijk, The Netherlands

e-mail: cecil.tranquille@esa.int

K. Hurley $\cdot$ H.S. Hudson

Space Sciences Laboratory, UC Berkeley, Berkeley, CA 94720, USA 
hard X-radiation predominantly at the chromospheric footpoints of coronal magnetic loops. Softer X-rays have thermal spectra with $k T=1-3 \mathrm{keV}$ and originate from the loops themselves. The soft X-ray emission lags in time according to the Neupert effect (Neupert, 1968; Dennis and Zarro, 1993). The duration of the hard X-ray emissions can vary from a few seconds to many tens of minutes and the photon energies can extend to the highest limit of the observations.

One of the scientific objectives of the GRB experiment flown on Ulysses (Hurley et al., 1992) was to make stereoscopic observations of solar flares from its unique high-latitude heliocentric orbit by comparison with data from other X-ray monitors flown on satellites in near-Earth or other interplanetary orbits (Hurley, 1986). Combining simultaneous measurements of solar X-rays from two or more satellites having different vantage points can provide useful information about the directivity of the emission as well as an estimate of the height of the emitting region in the photosphere. Deep-space observations contribute to the detection of flares occulted by the solar limb, a good method for isolating X-ray sources in the corona rather than at the chromospheric footpoints of the flare loop system (Frost and Dennis, 1971; Hudson, 1978; Kane et al., 1998).

The large aphelion distance of the Ulysses orbit $(\approx 5 \mathrm{AU})$, as well as the small size of the GRB detectors, helped to minimize saturation during the most energetic events. Detectors flown on spacecraft in orbits closer to the Sun can suffer from saturation and pulse pile-up effects at these times, resulting in reduced fidelity of the time profiles, especially around event maxima. This orbit also permitted almost continuous observations, as opposed to the regular eclipses due to Earth occultation in low Earth orbit, the usual site for solar hard X-ray observations.

Ulysses, although still functional, no longer routinely returns the basic data stream from GRB most suitable for solar hard X-ray observations. This is therefore an appropriate time to present the catalog of its observations, and that is the main function of this paper. We identify 59 GOES X-class flares observed simultaneously by GRB, and separately list another 14 comparable events for which the solar source was occulted by the solar disk as seen by Ulysses. The catalog of GRB information on these events is presented in tabular form in this paper, and also on-line as text and image files of the time histories. For the most energetic events there is substantial concern about pulse pile-up (as with many solar hard X-ray detectors; see Kane and Hudson, 1970 and Kane et al., 1980). Accordingly we have carried out a detailed comparison with RHESSI observations of a GOES X8.3 flare, as reported in Appendix A. We also describe Monte Carlo simulations of pulse pile-up in the GRB instrument in Appendix B; in general this is not as important an effect for GRB as it has been for other instruments for the reasons (great distance, small area) given above.

\section{The GRB Instrument}

The Ulysses Gamma-Ray Burst (GRB) instrument, has been described in detail elsewhere (Hurley et al., 1992). The two hard X-ray detectors are hemispherical shells of CsI(Tl) scintillators, with thickness $3 \mathrm{~mm}$ and diameter $51 \mathrm{~mm}$, coupled by plastic light-guides to photomultiplier tubes. The detectors are mounted externally on a $\approx 3 \mathrm{~m}$ boom. This remote mounting gives essentially all-sky coverage and also reduces the contamination from the radioactive source used by the spacecraft as a source of power throughout the mission (a radioisotope thermoelectric generator, or RTG). The two detectors together provide an approximately isotropic response, with a projected area of about $20 \mathrm{~cm}^{2}$ in all directions. They were continuously calibrated with a ${ }^{241} \mathrm{Am}$ source; energy spectra were read out on a daily 
basis to track the position and resolution of the $\approx 60 \mathrm{keV}$ line from this radioisotope. The day-to-day gain variations proved to be negligible, and the longer-term variations were compensated by analysis software and by adjusting the high voltage to the photomultiplier tubes. We were fortunate that Ulysses overlapped with RHESSI, and we have used a flare that was well observed by both instruments for a check on this calibration and other potential problems (Appendix A). The detector outputs are summed on board the spacecraft, and provide the integral counting rate over the spectral range $25-150 \mathrm{keV}$ with a time resolution of $0.25-2 \mathrm{~s}$, depending on the telemetry rate of the downlink. The background counting rate of the GRB instrument in this band is typically about 480 counts $\mathrm{s}^{-1}$; for solar observations this corresponds to $\approx 25$ photons $\mathrm{cm}^{-2} \mathrm{~s}^{-1}$ above $25 \mathrm{keV}$. A soft X-ray $(5-20 \mathrm{keV})$ detector comprising two Si sensors also formed part of the GRB instrument, but it became noisy during the unexpectedly long waiting period prior to launch and did not produce useful results. Spectral information in the hard X-ray range is available when the instrument automatically triggers into "burst mode" following a rapid increase in the detector count rate due to a cosmic gamma-ray burst or a solar flare, but we do not make use of this data mode for the catalog described in this paper. The instrument began observations in October 1990, and with the exception of a few periods, such as the Jupiter encounter in February 1992, it remained in continuous operation until November 2003. At this time, the RTG power became insufficient to keep all the experiments on. Prior to this, the data recovery from the mission was over 95\%, and the GRB experiment observed over 1500 solar flare X-ray events (a list may be found at http://helio.esa.int/ulysses/ftp/public/outgoing/grb/grb_solar_events.txt and in the on-line material for this paper).

For the biggest events the GRB data require corrections for dead time and for counter rollover. We base the dead time corrections on pre-launch calibrations. Figure 1 shows an example of the large X-ray event observed by GRB on 1 June 1991, that required correction for both rollover and dead time effects. The nominal energy resolution of GRB is $40 \%$ at $60 \mathrm{keV}$, reflecting its optimization for high counting rates rather than high resolution.

\section{Thermal vs. Nonthermal Hard X-Rays}

Solar hard X-ray spectroscopy presents particular observational problems because of the great dynamic range in source brightness. One of the most worrisome of these is pulse pile-up (Kane and Hudson, 1970; Datlowe, 1975), which we deal with in Appendix B. Inorganic scintillators such as the CsI(Tl) of Ulysses, or the CsI(Na) of SMM (Orwig, Frost, and Dennis, 1980), have good photopeak efficiencies but also relatively slow pulses, which exacerbates this problem.

In addition to this difficulty there is the problem of the intense soft X-ray spectrum. A solar flare may have a very large spectral intensity at a few $\mathrm{keV}$, in a spectrum with a steep exponential fall off ( $k T \approx 3 \mathrm{keV}$ ) to higher energies. Because scintillation counters have relatively poor energy resolution, pulses from this component can readily be confused with those from true hard X-rays. This "thermal feed-through" effect can be seen in most solar hard X-ray photometers, especially those with relatively thin entrance windows. It is systematically more important for flares of greater magnitude because of the general correlation of soft X-ray effective temperature with emission measure (Garcia and McIntosh, 1992; Feldman, 1996). For this reason events such as that of Figure 1 tend to show smooth "hard $\mathrm{X}$-ray" light curves, which for a fixed threshold show a relatively more thermal component. Note that this is independent of considerations of pulse pile-up effects and only depends upon the relative magnitudes of the thermal and non-thermal components. 


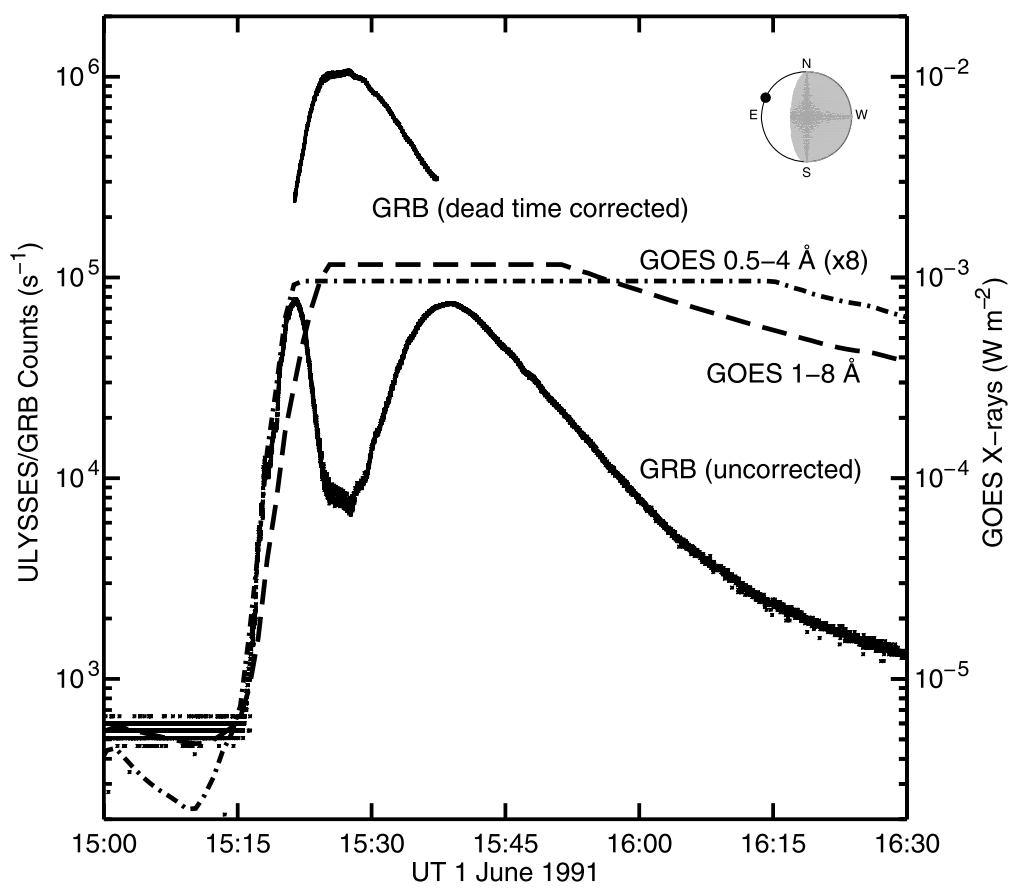

Figure 1 A solar flare measured by Ulysses GRB (1 June 1991, day 152; see Kane et al., 1995). The flare was classified as X12 from GOES measurements, and its location was on the east limb (N25E90) of the visible solar disk. The raw GRB count rate recorded by the instrument (after correction for buffer overflow) is plotted in the lower curve, with the deep minimum seen in the time profile due to dead time. The upper points show the true count rate with full corrections. The dashed and dot-dashed lines show the GOES XL (1-8 $\mathrm{A})$ and XS $(0.5-4 \AA)$ channels, respectively, both of which saturate for an event of this magnitude. The GOES times are corrected for light travel time to the position of Ulysses. The inset of the solar disk shows the view of the Sun from Earth, shaded for regions not visible from the position of Ulysses. The location of the flare is marked by a black dot.

High-resolution observations with Ge detectors (Lin et al., 1981), now carried out systematically with the Reuven Ramaty High-Energy Solar Spectrometric Imager spacecraft (RHESSI) (e.g., Holman et al., 2003), make it clear that this artefact complicates all solar hard X-ray catalogs derived from scintillation counters. Interestingly, it also makes it very difficult to recognize the analogous thermal/non-thermal components of stellar hard X-ray spectra because of their still higher thermal temperatures (Osten et al., 2007).

\section{Observations}

\subsection{GOES Events}

Information relevant to this study was extracted from the NOAA reports for all of the Xclass flares recorded by GOES since the launch of Ulysses in October 1990 until the GRB instrument was temporarily switched off in November 2003. Table 1 lists the 159 X-class flare events used in this report from the NOAA catalogs. The date of each event is provided, together with its start and end times (at GOES), and the time of the peak seen in the GOES 
Figure 2 Heliographic locations of all X-class flares observed by GOES over the GRB observing interval (October 1990 to late 2003).

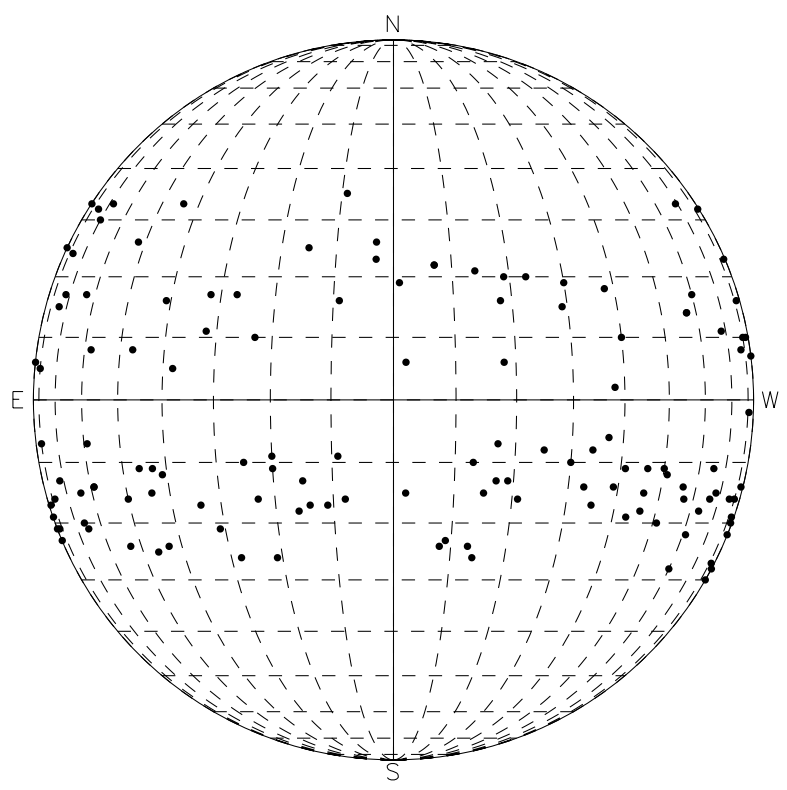

$\mathrm{X}$-ray time profile. In addition, the flare location (solar longitude and latitude) and peak XL $(1-8 \AA)$ flux are given. From the position of the Ulysses spacecraft (radial distance, solar longitude and latitude, also tabulated), we calculate whether the GOES flare should have been visible or not from the viewpoint of Ulysses. This is done by determining the angle (also given in Table 1) between the position of Ulysses, the center of the Sun, and the location of the flare on the solar surface. If this angle is between $0^{\circ}$ and $90^{\circ}$, then the flare is on the hemisphere of the Sun visible to Ulysses. An angle of $180^{\circ}$ represents a point on the hidden hemisphere of the Sun, directly opposite to the position of Ulysses. For 18 events, the location of the flare on the solar surface is not known from the NOAA reports, so it is not possible to determine unambiguously whether they could be seen by GRB or not.

Figure 2 displays the locations of the X-class flares on the visible hemisphere of the Sun (as seen from Earth) using the data from the SGAS reports. As is well known (Smith and Smith, 1963), flares cluster around mid-latitude bands north and south of the solar equator. There can also be significant asymmetry in the number of flares seen in the two hemispheres as discussed for example by Garcia (1990).

A significant concurrent increase in the GRB count rate (Y in Table 1) was required to confirm that each GOES X-class event calculated to be visible from the position of Ulysses, was indeed seen (a non-zero peak GRB count rate). Of the 65 events seen by GOES that should also have been detected by Ulysses, 59 events registered a count rate increase in the GRB instrument as expected. A single event (29 May 1991, GOES class X1) showed no signature in the count rate profile, possibly due to the combination of a steep hard $\mathrm{X}$-ray spectrum and the relatively large radial distance (3.17 AU) of Ulysses. A further three events showed no apparent signature in the GRB data due to a high instrument background resulting from contamination by in situ solar protons. Two events could not be verified at all because of data gaps in the GRB telemetry. Thus the Ulysses GRB data are consistent with the hypothesis that an X-class flare always has a hard, non-thermal emission component, which in most cases extends well above $25 \mathrm{keV}$. 


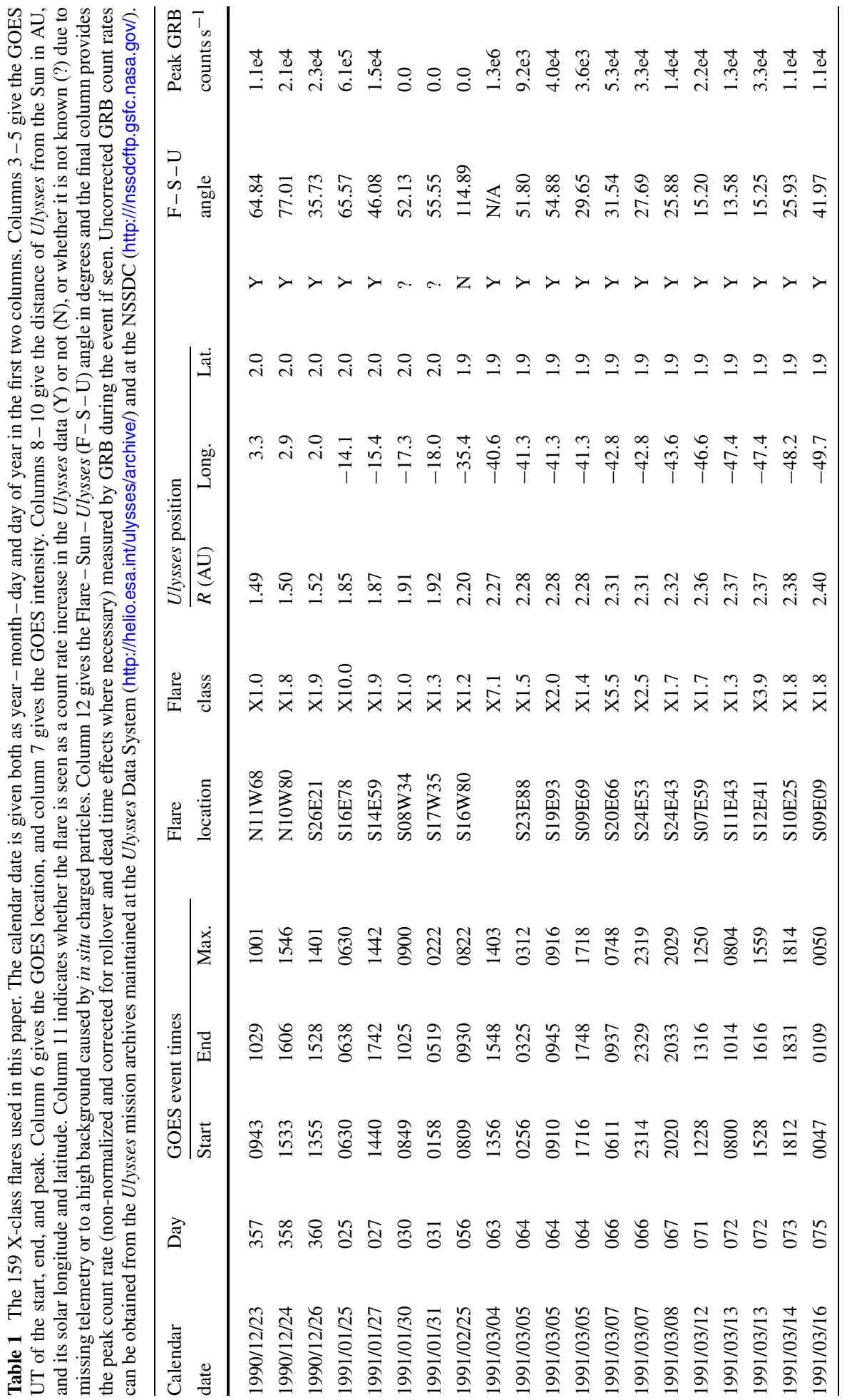




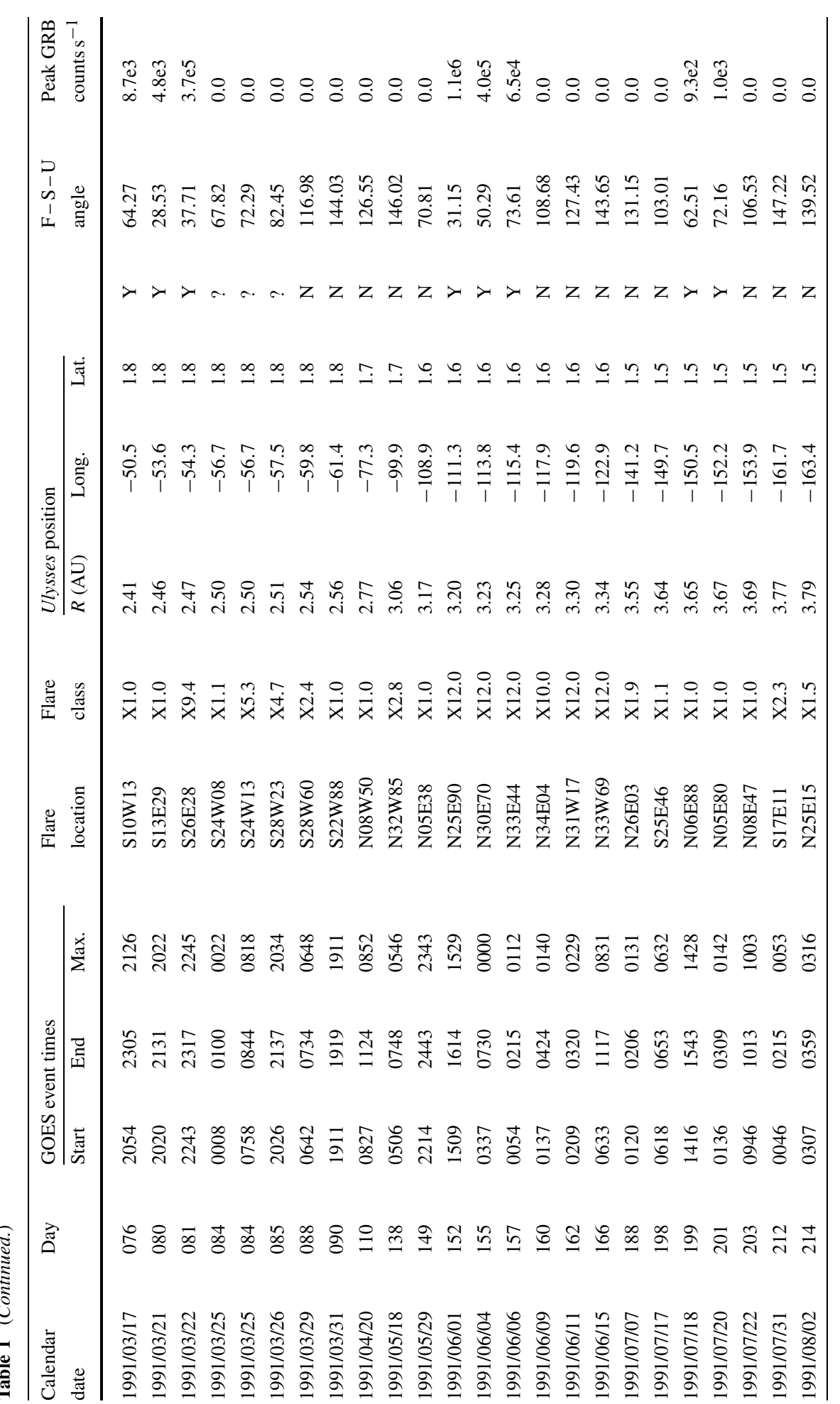




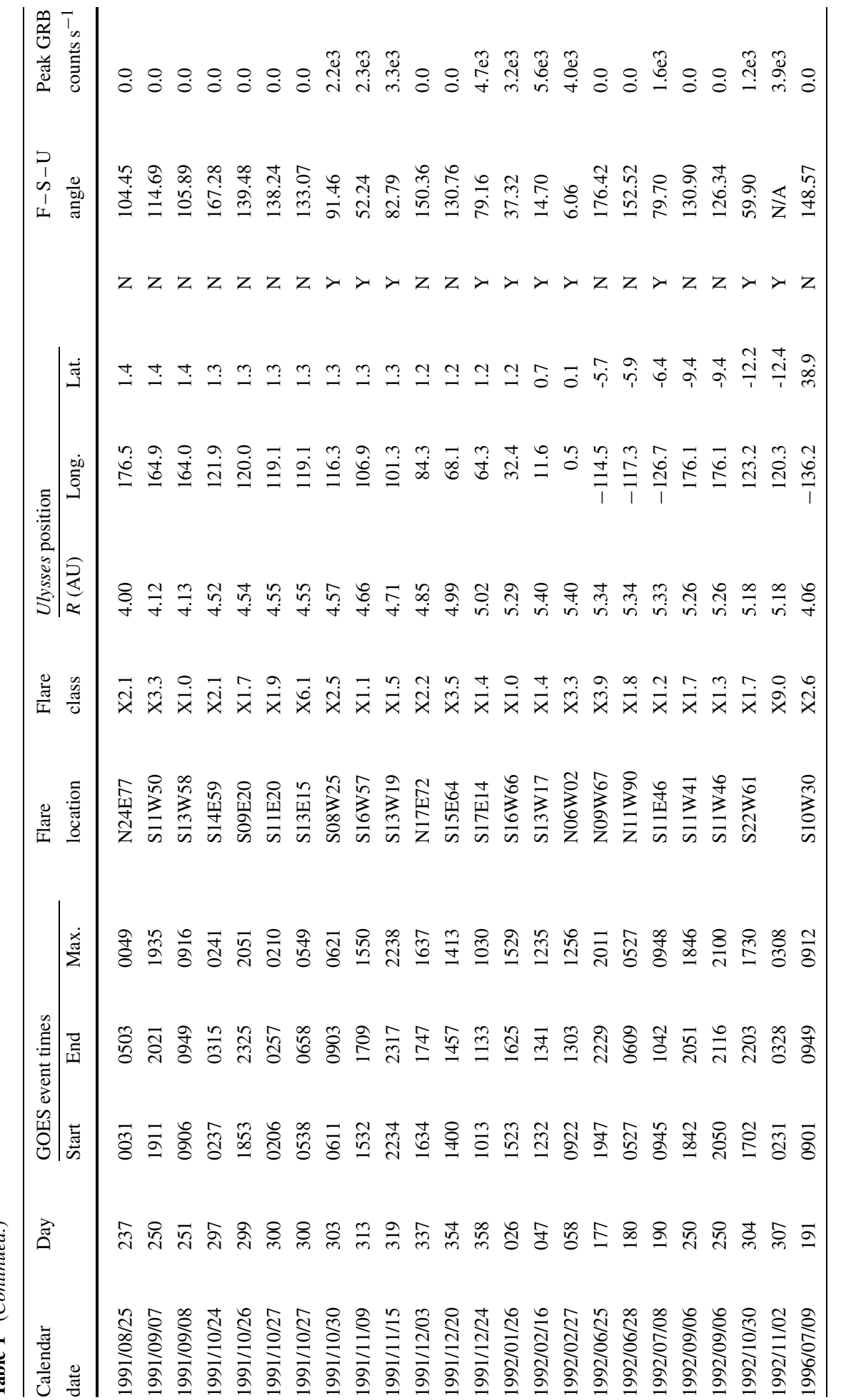




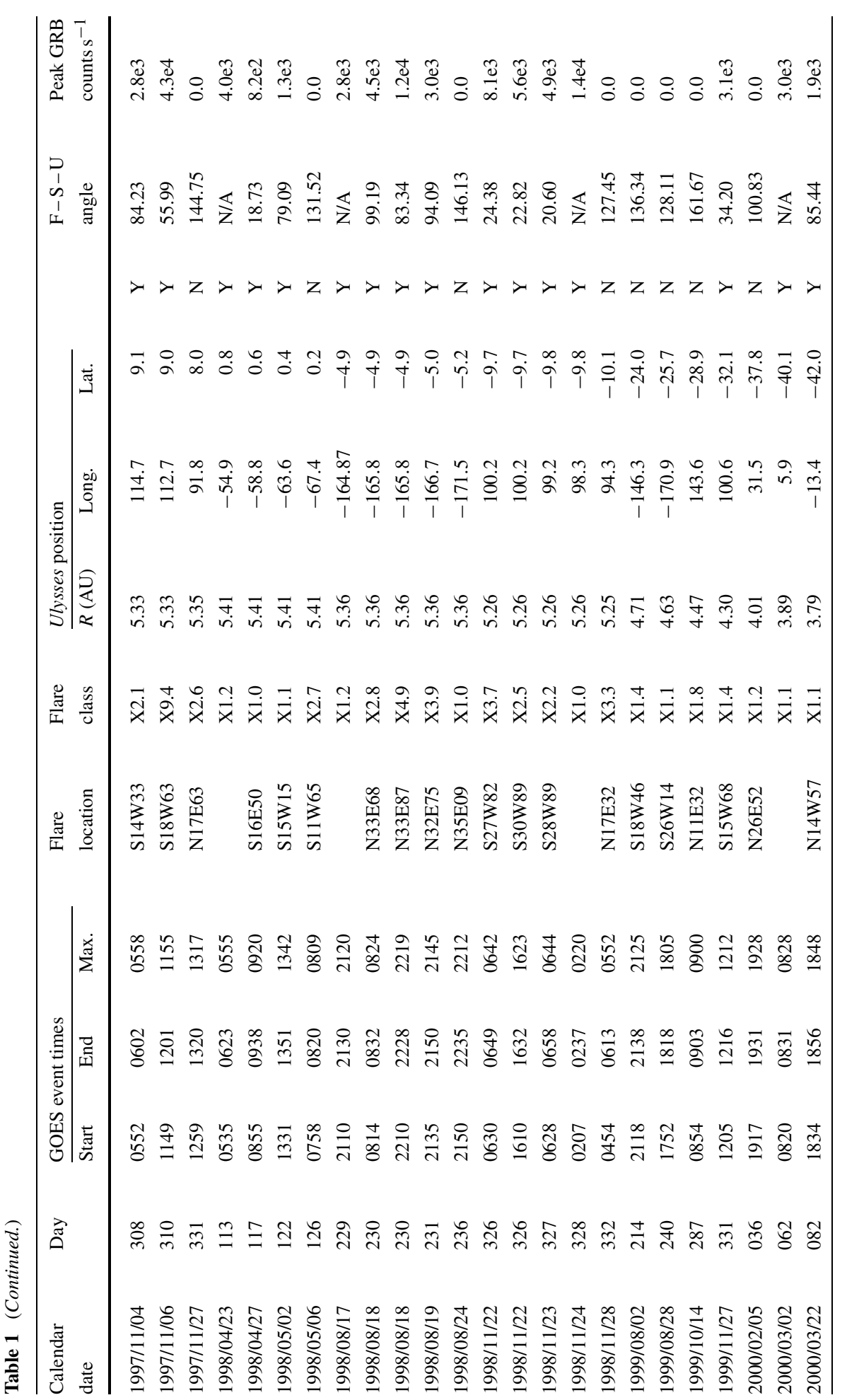




\begin{tabular}{|c|c|}
\hline 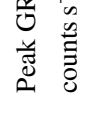 & 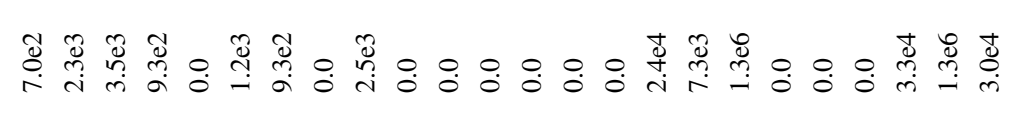 \\
\hline $\begin{array}{ll}1 & 0 \\
1 & \frac{0}{600} \\
1 & =\end{array}$ & 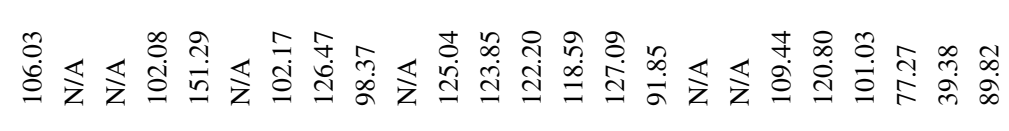 \\
\hline & $\succ \succ \succ \succ z \succ \succ z \succ z z z z z z \succ \succ \succ z z z \succ \succ \succ$ \\
\hline 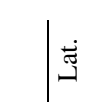 & 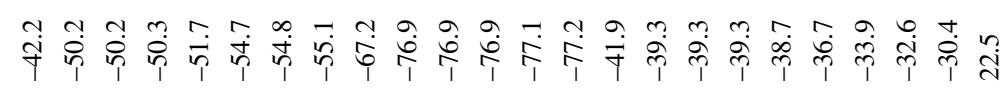 \\
\hline$\stackrel{\text { ij }}{\overline{0}}$ & 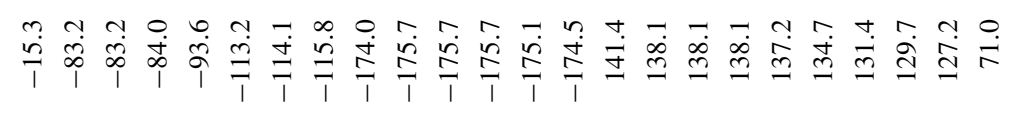 \\
\hline $\overrightarrow{5}$ & 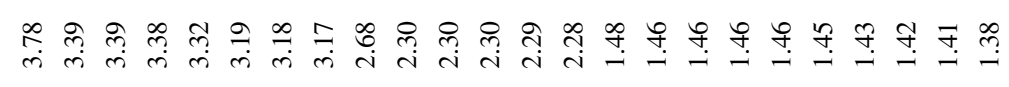 \\
\hline $\begin{array}{l}\frac{\mathscr{D}}{\tilde{I}} \\
\frac{\mathscr{L}}{I} \\
\frac{\sigma}{0}\end{array}$ & 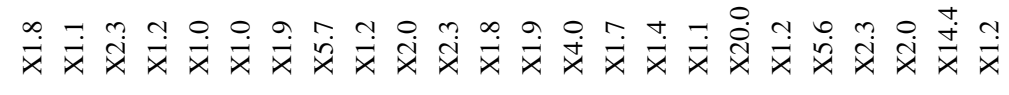 \\
\hline 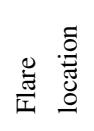 & 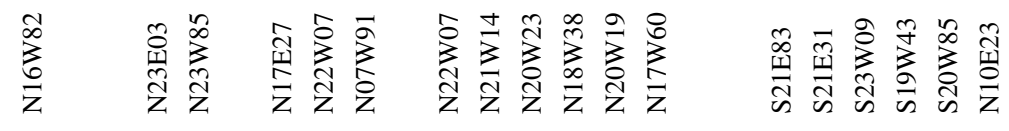 \\
\hline 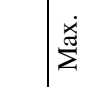 & 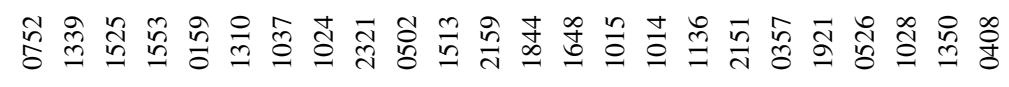 \\
\hline 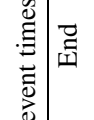 & 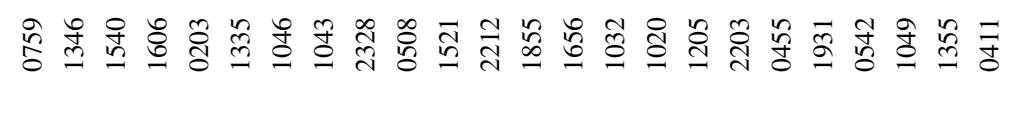 \\
\hline 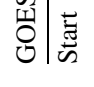 & 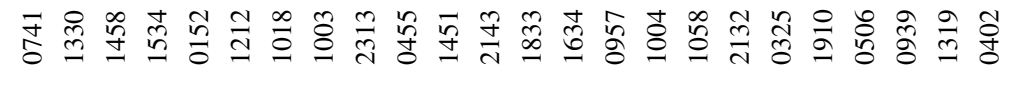 \\
\hline$\vec{\nexists}$ & 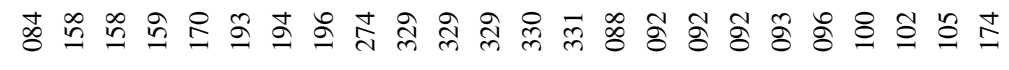 \\
\hline 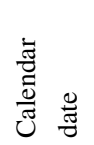 & 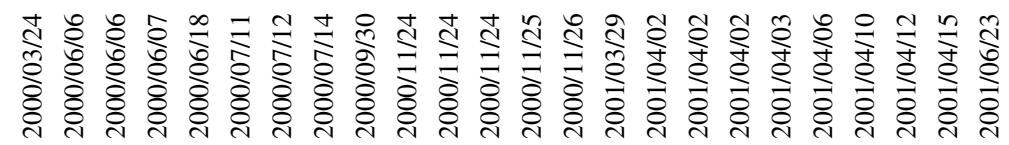 \\
\hline
\end{tabular}




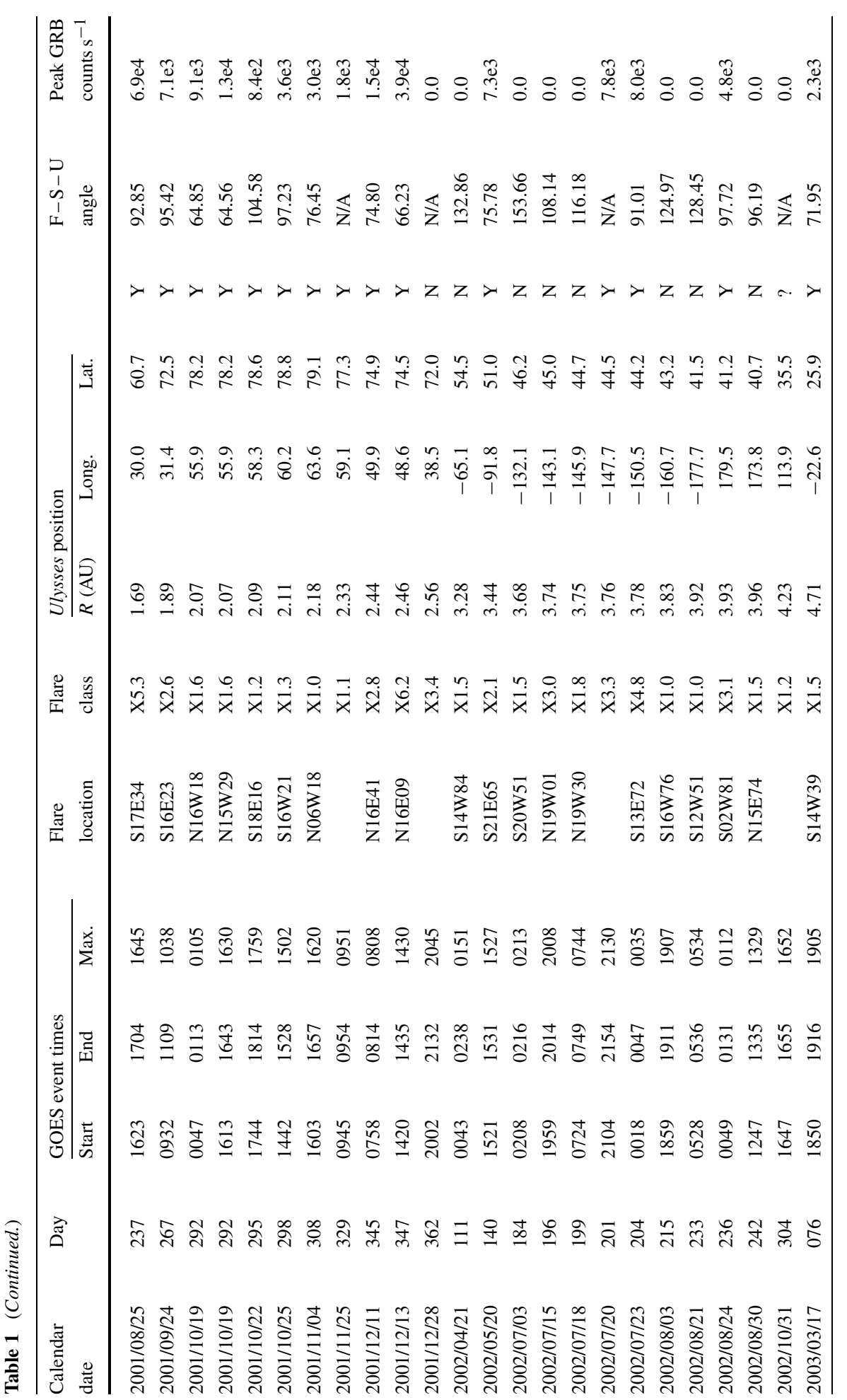




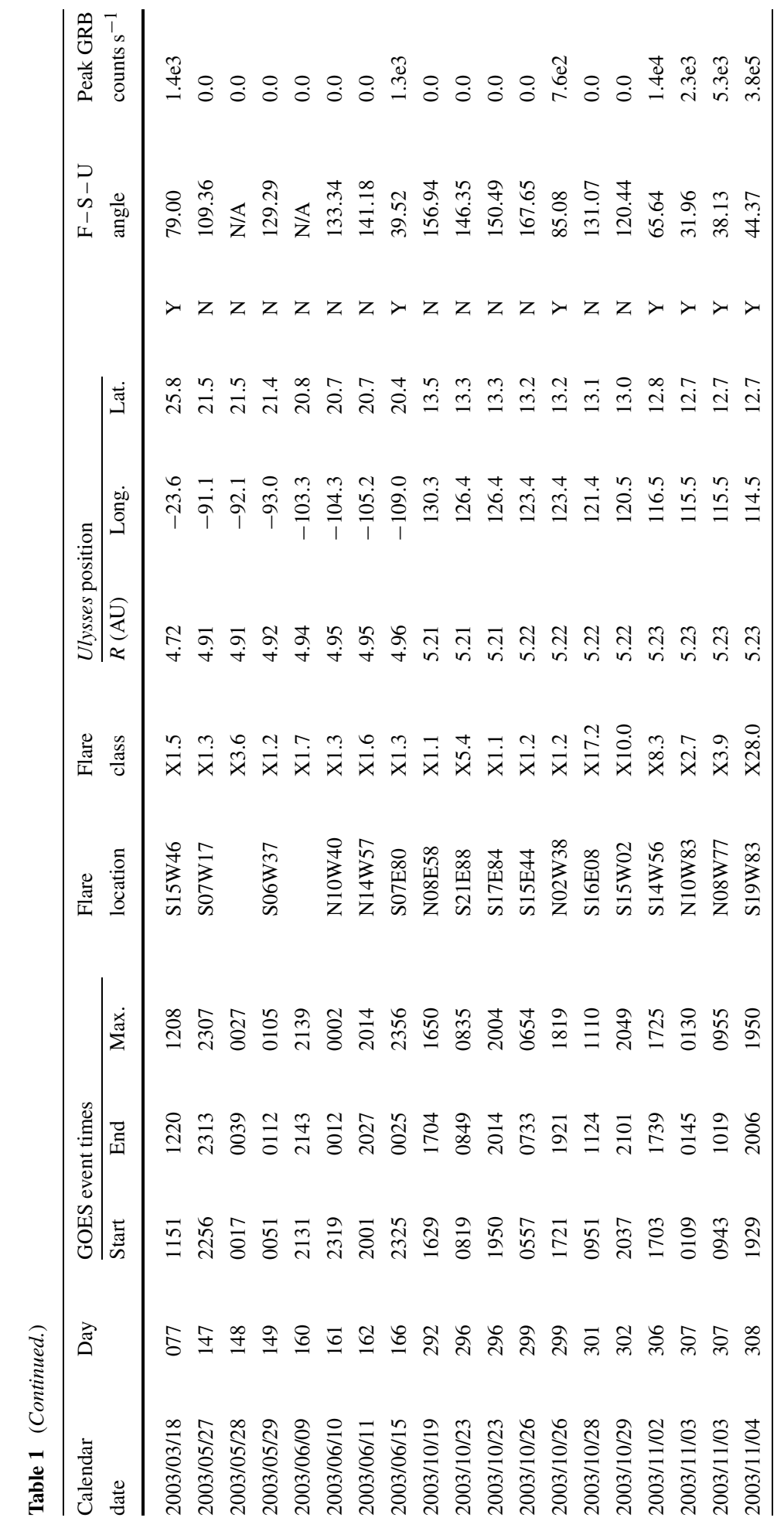




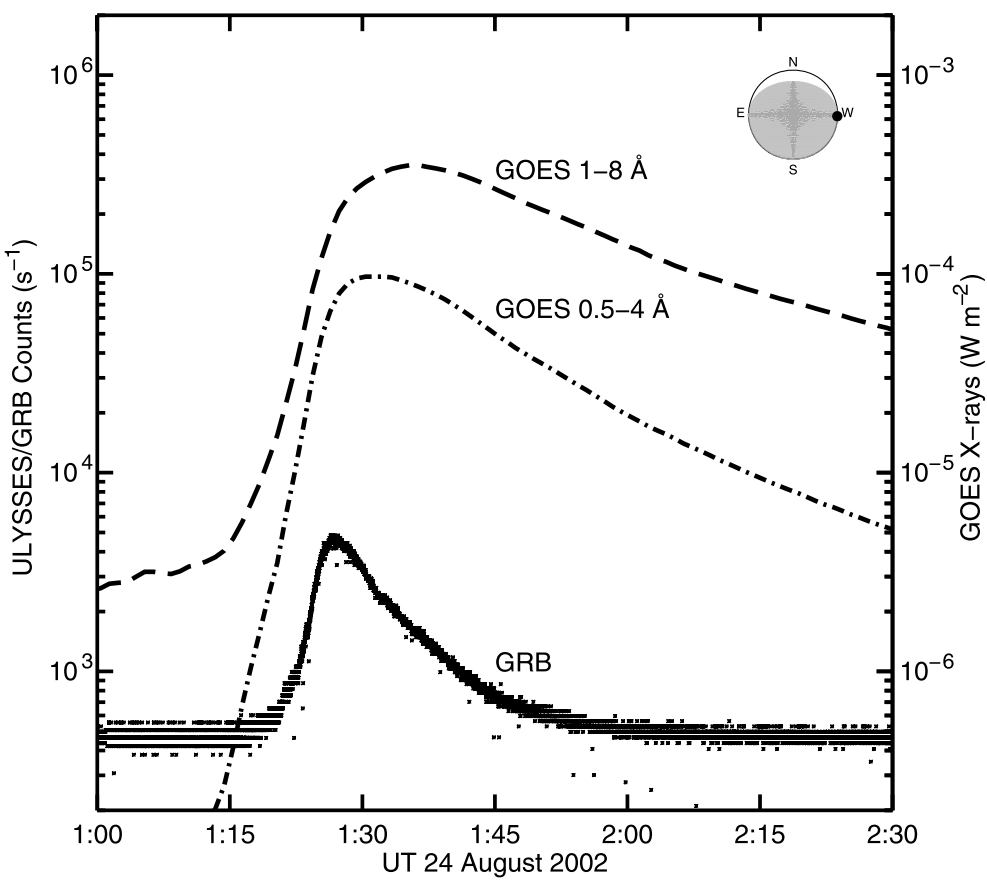

Figure 3 An example of an X3.1 flare measured by GOES that was behind the solar limb as viewed from the orbit of Ulysses. The hemisphere shown is that seen from the Earth and the flare is located at S02W81. The unshaded region shows the part of this hemisphere which Ulysses was able to see at the time of the flare (24 August 2002), when the spacecraft was at heliographic coordinates (179.5W, 41.2N). The flare was located on the hidden hemisphere of the Sun, as viewed from Ulysses, but was sufficiently close to the solar limb to register X-ray photons in the GRB detector.

In addition to the flares predicted to be visible from the orbit of Ulysses, there were another 14 flares which were recorded by GRB from behind the solar limb. The Ulysses-Sunflare angle for these events ranged from $90^{\circ}$ to no more than $110^{\circ}$. Figure 3 is an example of such a flare, showing the relative location of the flare site to the solar limb. Note that the $\mathrm{N}$ or $\mathrm{S}$ limb may be involved in the flare occultation as viewed from Ulysses, rather than the predominant E or W limb as viewed from the Earth. Figures showing the GRB responses to the 63 flares directly visible to GRB and the 14 flares occurring behind the limb (as seen from Ulysses) may be found at http://helio.esa.int/ulysses/ftp/public/outgoing/grb/table1/ and in the on-line material for this paper.

\subsection{Scaling Law}

The 59 common events seen by both GOES and GRB were analyzed to establish a correlation between the intensity assigned to the flares in the SGAS reports, and the maximum GRB count rate after normalization for the radial distance of Ulysses (Figure 4). Excluding the data points for the two largest events (circles) seen by GRB (the event shown in Figure 1 and the X28 event of 4 November 2003), we find a power-law fit through the common events (squares) measured by both GOES (peak energy flux $F_{\mathrm{GOES}}, 10^{-4} \mathrm{~W} \mathrm{~m}^{-2}$, or X1 units) and 


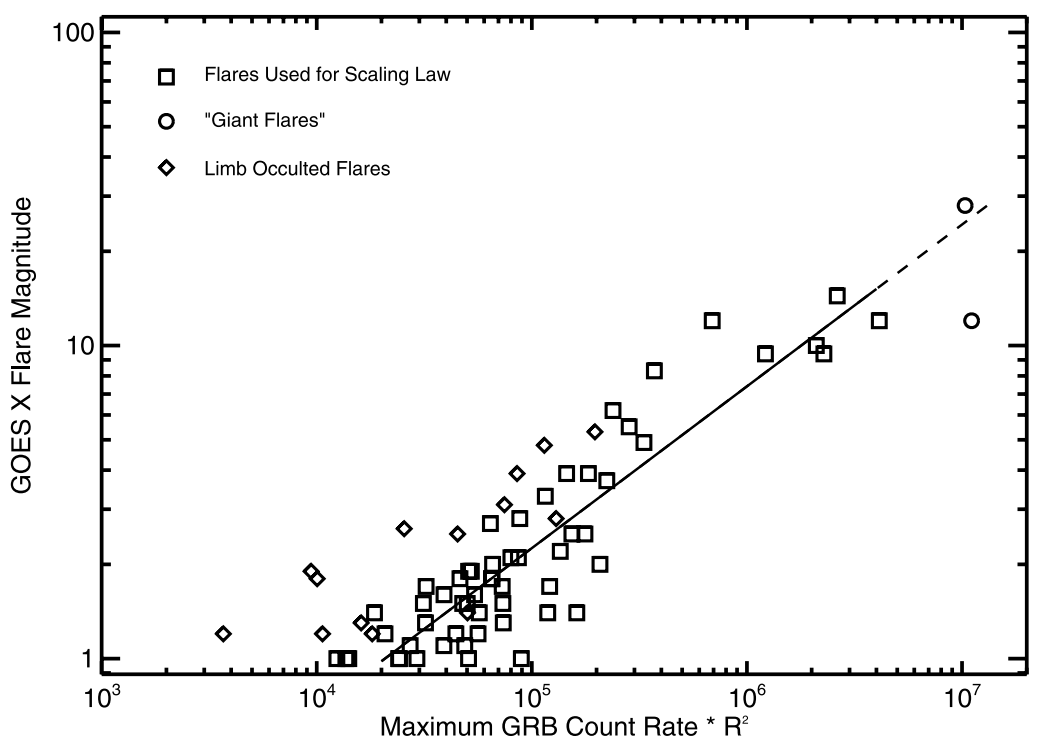

Figure 4 X-class flares seen by both GOES and GRB are used to establish the scaling law between the normalized maximum count rate registered by GRB and the intensity assigned to the flare from GOES measurements. The 59 flares fully visible to both GOES and GRB were used to obtain the fit (squares), excluding the two most intense flares measured by GRB (circles), one of which was the X28 event of 4 November 2003 , and the other the event of 1 June 1991 shown in Figure 1. The 14 flares seen from behind the solar limb from the viewpoint of Ulysses are also plotted (diamonds), although they are not used in the fit.

GRB (peak count-rate excess $F_{\mathrm{GRB}}$, counts s ${ }^{-1}$ );

$$
F_{\mathrm{GOES}}=a\left(F_{\mathrm{GRB}}\right)^{b}
$$

and we find $a=0.006 \pm 0.002$ and $b=0.52 \pm 0.03$. If the seven most intense events are excluded from the fit, values for $a$ and $b$ change slightly to 0.005 and 0.54 , respectively. The fit also excludes data for the 14 behind-the-disk events (diamonds) because of the bias due to partial occultation of the GRB source. Indeed, these events lie well to the left (thus a lower normalized GRB count rate) of events with similar GOES flare classification seen directly on the hemisphere visible to Ulysses. The derived scaling law is significantly non-linear. This could be the result of systematic error in either (or both) of the data sets, or it could reflect the presence of new mechanisms found in X-class flares but not in less energetic ones. The non-linearity does not reflect selection bias, since selection via the GOES data has given us a complete sample.

This scaling law deviates from the rough proportionality expected from other observations, in particular the slope 0.83 found by Battaglia, Grigis, and Benz (2005) for a comparison of GOES with the RHESSI $35 \mathrm{keV}$ spectral flux. We believe RHESSI to be the most definitive because its spectral resolution permits it to isolate the non-thermal component more cleanly than previous instruments based on scintillation counters. Our correlation result, $b \approx 0.5$, differs strikingly from the RHESSI result. We interpret this as evidence for systematic "thermal feed-through" into the $>25 \mathrm{keV}$ GRB counting rates. The departure from proportionality is in the sense expected from the weak statistical correlation found between GOES temperature and emission measure, as discussed in Section 3. In Appendix A, the GRB thermal feed-through is shown to be about a factor of 2 for an X8.3 flare. 
Assuming exact proportionality and fitting only to the events below GOES X10, we find $F_{\mathrm{GOES}}=A F_{\mathrm{GRB}}$, with $A=(3.0 \pm 0.5) \times 10^{-5} \mathrm{X} 1$ units per GRB count s $\mathrm{s}^{-1}$.

\subsection{The Most Energetic Events}

During the last (Cycle 22) and the present (23) solar cycles, Ulysses GRB successfully recorded the most energetic solar flares (Kane et al., 1995), among which the major flare of 4 November 2003, was the most notable (Kane, McTiernan, and Hurley, 2005). Kane, McTiernan, and Hurley (2005) and others have used the term "giant flare" to describe these top few flares, typically at GOES level X10 or above; they speculated that such flares utilize the bulk of the magnetically stored energy. We do not now regard a "giant flare" as a physically distinct category and believe that many of the reported properties of these events result from the thermal feed-through mechanism described in Section 3 and in Appendix A. These events saturate GOES and we have used the GRB response as a check on the effective GOES classification of the 4 November 2003 event, one of the most energetic flares on record. An extrapolation of the GOES X-ray count rates during the 15 minutes of detector saturation was consistent with a flare classification greater than X28 (Kiplinger and Garcia, 2004). Separate studies using the phase of VLF radio signals propagated in the ionosphere (Thomson, Rodger, and Dowden, 2004), and the measurement of the ionospheric attenuation of the galactic radio background (Brodrick, Tingay, and Wieringa, 2005), yielded higher estimates of the peak flux, as high as X40.

We use the scaling law derived in the previous section to estimate a flare class of $\mathrm{X} 24.8 \pm 12.6$ from the normalized peak count rate recorded by GRB at 20:34 UT on 4 November 2003 (Figure 5); this uncertainty, derived from the scaling law, is greater than the photometric calibration of GRB, which we estimate at 10-20\% (see Appendix A). This result is consistent with the value obtained by a smooth extrapolation of the GOES data when the X-ray sensor saturated. From GRB measurements, the 4 November 2003 flare was slightly exceeded by the flare on 1 June 1991 (Figure 1), which was located at latitude $25^{\circ} \mathrm{N}$ and probably about $15^{\circ}$ behind the east limb based on the locations of subsequent X-class flares from the same active region (NOAA 6659) once it rotated onto the disk visible from Earth. This flare was only classified as an X12 event by GOES, but it is quite likely that this was a much larger event which was significantly underestimated due to its probable occultation by the solar limb as viewed from Earth. According to the study of Tomczak (2001) the depth of occultation in this case was great enough to obscure the footpoint regions of the flare. Because the GRB flux exceeded that expected from the correlation, we suggest retrospectively that this flare also belongs in the same category, with effective GOES class above X20. Such large events are few in number and at the limit of our scaling law, so this conclusion is speculative, but we note the event of 30 March 1969 (Frost and Dennis, 1971, also deeply disk-occulted) as another possible example.

\subsection{Occurrence Distribution Function}

The GRB observations provide the most extensive and homogeneous view of solar hard $\mathrm{X}$-ray events to date, comparable in length to that of the Solar Maximum Mission (Dennis, 1988), but with a better duty cycle and a dynamic range that favors (because of small detector area and distant orbit) the most energetic events. As noted by Hudson (1991), the distribution function of flare energies follows such a flat power law that it would diverge at large energies, so that an upper limit or at least a roll-over of the distribution is necessary. The GRB database gives us an opportunity to define the occurrence frequency of the most energetic events 


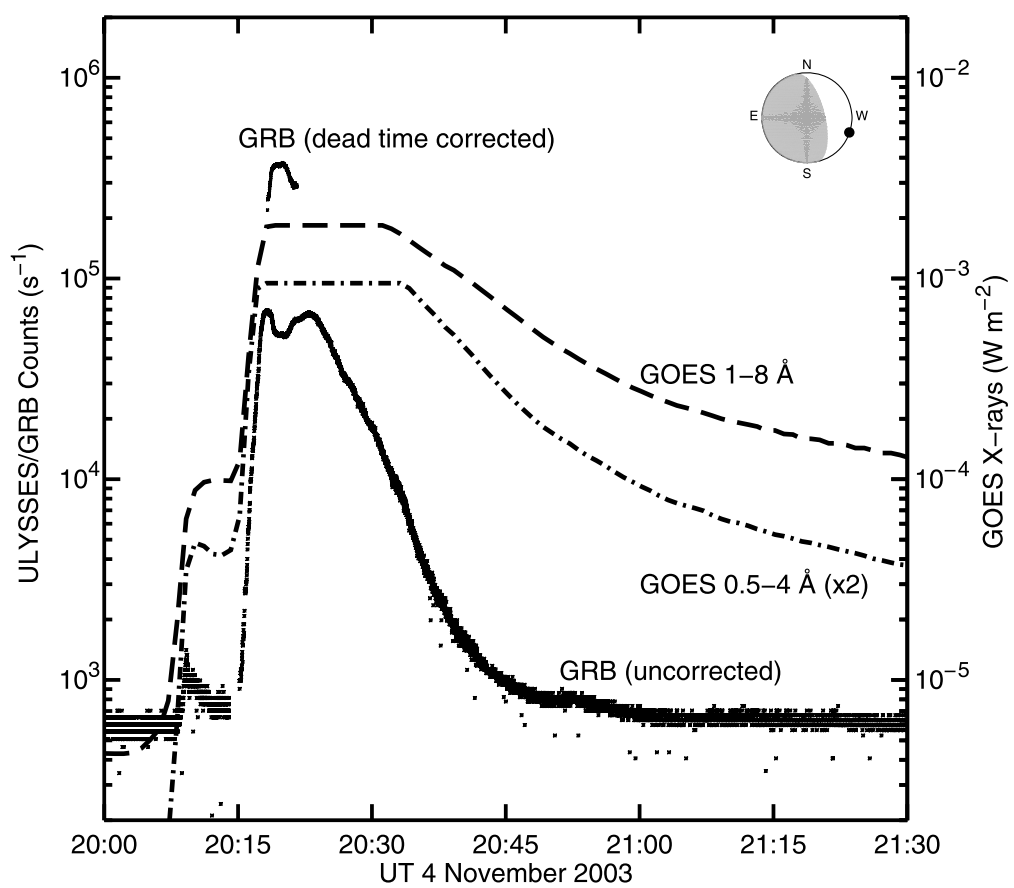

Figure 5 The response of the GRB instrument during the X28 flare on 4 November 2003, which was located at S19W83 on the visible solar disk. The large radial distance of Ulysses from the Sun allowed this flare to be monitored throughout its duration without significant saturation of the X-ray photon detectors.

(i.e., out to the two major flares discussed above) and to investigate whether the distribution follows the expected flat power law.

Figure 6 shows the integral distribution for the 59 well-observed events of Table 1 with accompanying GOES events. We fit this with a simple power-law and find no evidence for a cutoff, in contrast to Kucera et al. (1997) who established probabilities for a highenergy rollover in the flare frequency distribution from HXRBS measurements on SMM. On the basis of the number of events in our complete sample, it would be necessary to observe for several solar cycles to improve on this conclusion, or else to use proxy data that contain a longer historical record (e.g., Reedy and Marti, 1991). There is good evidence from cosmic-ray proxies for a steepening of the distribution of solar energetic particle fluences (Lingenfelter and Hudson, 1980; Reedy, 1996).

The distribution in Figure 6 shows a roll-over towards lower event magnitudes, which we attribute to the sensitivity limit for this sample, and the fits below refer to the range above $5 \times 10^{4} \mathrm{GRB}$ counts $\mathrm{s}^{-1}$ normalized to one AU. This provides a sample of 41 events over the range X2-X25 according to our scaling law. As with previous hard X-ray studies at lower event energies show (see, e.g., Crosby et al. 1993, 1998), a power-law with an index flatter than -2.0 fits reasonably well. We write the differential distribution $\mathrm{d} N / \mathrm{d} S \propto S^{\alpha}$ and use a Monte Carlo method to fit the slope of the integral distribution (as shown in Figure 6) and its errors. This yields $\alpha=-1.61 \pm 0.04$ for the 41 events above the clear knee in the distribution, which we attribute to selection bias. If we exclude the nine events with highest GRB counts, including the two most energetic events, the slope changes to $-1.75 \pm 0.08$. Finally, we have done a direct fit to the differential distribution, using the 


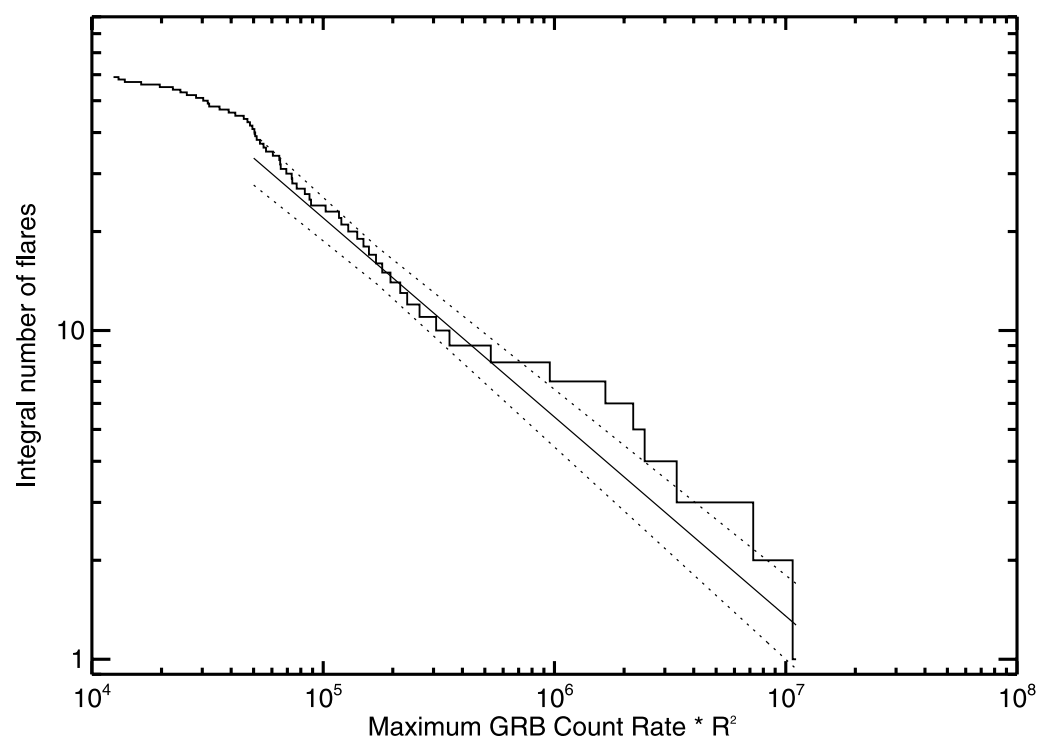

Figure 6 Integral distribution of normalized maximum GRB count rates, selecting events with known GOES occurrence. The solid line shows the best-fit slope of $1.61 \pm 0.04$ for the differential distribution, and the dotted lines give an approximate range of uncertainties.

maximum-likelihood method of Crawford et al. (1970), and find $\alpha=-1.72$. These results are roughly consistent with values obtained for less energetic hard X-ray events (Crosby et al., 1998 found $1.732 \pm 0.008$ from the WATCH experiment). Some studies of soft $\mathrm{X}$-ray events have found steeper slopes (e.g., Veronig et al., 2002) but the slopes may not be directly comparable.

\subsection{Loop Height Estimates}

Events located behind the solar limb will be partially occulted, but may nevertheless be visible if the loop height is sufficiently large. The number of GOES X-class flares (with known solar location) was accumulated in ten-degree bins of the angle between Ulysses and the flare site. Only events from the complete list in Table 1 for which the GRB instrument registered valid measurements (no contamination of the X-ray detectors by solar particles, and nominal instrument telemetry) have been used.

All but one of the 11 partially occulted flares up to $10^{\circ}$ behind the solar limb are visible to the GRB detectors. Between $10^{\circ}$ and $20^{\circ}$ behind the solar limb, a third of the 12 flares that occurred are visible. The event of 24 March 2000, is the most extreme example, at an angle of $106^{\circ}$. At such an angle the occultation height (the source height, assuming a radial structure) would be $0.04 R_{\odot}$ or about $3 \times 10^{4} \mathrm{~km}$. This height is consistent with earlier observations made with combined hard X-ray and microwave imaging of flare loops described by Nakajima et al. (1985) and Cliver et al. (1986).

\subsection{A Catalog of Far-Side Events}

The X-class flares listed in Table 1 that were measured by both GRB and GOES do not constitute an exhaustive record of intense X-ray events throughout the Ulysses mission. 
There are many more signatures in the GRB data that correspond to X-class flares not seen by GOES, implying that the flare sites must be located on the far-side of the Sun from the viewpoint of the Earth.

Table 2 contains a separate list of these flares, providing the date and time of each event, together with the effective GOES X-ray class estimated using the scaling law derived above in Section 4.2. The criteria used to identify these flares were a GRB intensity profile consistent with a solar flare event, a normalized peak count rate greater than a threshold value of $2 \times 10^{4}$ counts s $^{-1}$ and the absence of any M- or X-class flare in the GOES event list. Although it is usually not possible to precisely locate the flare sites for these events, the timing of each GRB event corresponds to a range of potential solar longitudes and latitudes from the known viewing angle of Ulysses. Figures showing the GRB response during these inferred far-side events may be found at http://helio.esa.int/ulysses/ftp/public/outgoing/grb/table2/ and in the on-line material.

As expected from the GOES distribution function, the majority of the 82 far-side events thus selected were smaller X-class events. However, there was one inferred X15 event recorded during the intense period of solar activity in 1991. During certain periods of the Ulysses orbit, the combination of GRB and GOES measurements provided almost uninterrupted full-Sun coverage of major solar X-ray flare activity.

\section{Discussion and Conclusions}

The Ulysses data provide a unique stereoscopic view of solar hard X-ray emission and complement other observations in different ways. In this paper we have summarized the entire data set of energetic (GOES class $>$ X1.0) events to date, a 13-year span that covers the maximum of Carrington cycle 23. This represents only about $10 \%$ of the full Ulysses GRB solar event list. We present the entire list of X-class flares observed during this time interval after establishing a correlation of the GRB hard X-ray fluxes with the GOES soft X-ray fluxes, which we summarize in the scaling law defined above. This scaling enables us to confirm the magnitude of the 4 November 2003 event as obtained by a simple extrapolation of the saturated GOES light curve at about X25, thus calling into question the somewhat larger fluxes suggested by the ionospheric data. We find the 1 June 1991 event to have had a comparable magnitude despite a deep occultation, and suggest that it was comparable in magnitude to the 4 November 2003 event, of GOES class X20 or higher.

The Ulysses GRB data are consistent with the hypothesis that all GOES X-class flares have hard non-thermal components which extend into the energy range above $25 \mathrm{keV}$. Our analysis indicates that the $25 \mathrm{keV}$ threshold of the GRB allows some "thermal feed-through" for the largest events, causing the correlation of GOES magnitude and peak GRB flux to deviate from the near-proportionality found in the RHESSI data. This finding is consistent with the known weak correlation of soft X-ray emission measure and effective temperature, such that more energetic events have larger thermal contributions in the hard X-ray band for a detector with poor spectral resolution. The occurrence frequency distribution continues with the expected flat power law to the greatest events observed (specifically, the two events mentioned above). There is thus no evidence for a cutoff within this sample.

Acknowledgements $\mathrm{KH}$ is grateful for Ulysses support under JPL Contracts 958056 and 1268385. The work of HSH was supported by NASA under grants NAS 5-98033 and NAG5-12878.

Open Access This article is distributed under the terms of the Creative Commons Attribution Noncommercial License which permits any noncommercial use, distribution, and reproduction in any medium, provided the original author(s) and source are credited. 
Table 2 Solar flare X-ray events (totaling 82) observed by Ulysses which were not observed by GOES, and which are presumably on the far-side of the Sun. Columns $1-3$ give the year, day of year, and UT in hours and minutes (at Ulysses). Column 4 gives the distance of Ulysses from the Sun in AU. Column 5 gives the non-normalized maximum GRB count rate after correction for dead time and rollover, and columns 6 and 7 give the inferred GOES X-class intensity and its uncertainty, derived from the scaling law in Section 4.2.

\begin{tabular}{|c|c|c|c|c|c|c|}
\hline Year & Day & $\begin{array}{l}\text { GRB } \\
\text { time }\end{array}$ & $\begin{array}{l}\text { Ulysses } \\
R(\mathrm{AU})\end{array}$ & $\begin{array}{l}\text { Peak GRB } \\
\text { counts s }{ }^{-1}\end{array}$ & $\begin{array}{l}\text { Inferred } \\
\mathrm{X} \text {-class }\end{array}$ & $\begin{array}{l}+/- \\
\text { Error }\end{array}$ \\
\hline 1991 & 62 & 1901 & 2.26 & $5.5 \mathrm{e} 4$ & 3.8 & 2.0 \\
\hline 1991 & 118 & 1938 & 2.86 & $6.0 \mathrm{e} 3$ & 1.6 & 0.8 \\
\hline 1991 & 119 & 1416 & 2.87 & 4.6e 4 & 4.5 & 2.4 \\
\hline 1991 & 141 & 1349 & 3.09 & $5.0 \mathrm{e} 3$ & 1.5 & 0.8 \\
\hline 1991 & 145 & 1815 & 3.13 & $4.2 \mathrm{e} 3$ & 1.4 & 0.7 \\
\hline 1991 & 147 & 1518 & 3.15 & $3.7 \mathrm{e} 3$ & 1.3 & 0.7 \\
\hline 1991 & 150 & 0142 & 3.18 & $5.0 \mathrm{e} 3$ & 1.6 & 0.8 \\
\hline 1991 & 151 & 0524 & 3.19 & $1.9 \mathrm{e} 4$ & 3.2 & 1.7 \\
\hline 1991 & 171 & 2206 & 3.39 & $2.5 \mathrm{e} 3$ & 1.2 & 0.6 \\
\hline 1991 & 172 & 0901 & 3.40 & $4.9 \mathrm{e} 4$ & 5.5 & 3.0 \\
\hline 1991 & 172 & 1247 & 3.40 & $8.7 \mathrm{e} 3$ & 2.3 & 1.2 \\
\hline 1991 & 177 & 1606 & 3.44 & $2.7 \mathrm{e} 4$ & 4.1 & 2.2 \\
\hline 1991 & 177 & 2031 & 3.44 & $3.6 \mathrm{e} 5$ & 15.6 & 9.4 \\
\hline 1991 & 179 & 1449 & 3.46 & $3.0 \mathrm{e} 4$ & 4.4 & 2.4 \\
\hline 1991 & 286 & 2320 & 4.43 & $2.8 \mathrm{e} 3$ & 1.7 & 0.8 \\
\hline 1991 & 287 & 1543 & 4.44 & $4.9 \mathrm{e} 3$ & 2.2 & 1.1 \\
\hline 1991 & 304 & 1103 & 4.58 & $2.0 \mathrm{e} 3$ & 1.4 & 0.7 \\
\hline 1991 & 305 & 0824 & 4.59 & $2.0 \mathrm{e} 3$ & 1.5 & 0.7 \\
\hline 1991 & 305 & 2013 & 4.59 & $2.0 \mathrm{e} 3$ & 1.4 & 0.7 \\
\hline 1992 & 5 & 0816 & 5.12 & $2.0 \mathrm{e} 3$ & 1.6 & 0.8 \\
\hline 1992 & 9 & 2028 & 5.15 & $1.3 \mathrm{e} 3$ & 1.3 & 0.7 \\
\hline 1992 & 13 & 0730 & 5.18 & $2.0 \mathrm{e} 3$ & 1.6 & 0.8 \\
\hline 1992 & 243 & 0859 & 5.27 & $1.3 \mathrm{e} 3$ & 1.3 & 0.7 \\
\hline 1992 & 245 & 0112 & 5.27 & $1.1 \mathrm{e} 3$ & 1.2 & 0.6 \\
\hline 1992 & 263 & 0754 & 5.25 & $2.8 \mathrm{e} 3$ & 2.0 & 1.0 \\
\hline 1992 & 280 & 1119 & 5.22 & $2.0 \mathrm{e} 3$ & 1.6 & 0.8 \\
\hline 1993 & 171 & 1516 & 4.62 & $1.3 \mathrm{e} 3$ & 1.2 & 0.6 \\
\hline 1993 & 269 & 0139 & 4.26 & $2.3 \mathrm{e} 3$ & 1.4 & 0.7 \\
\hline 1995 & 115 & 2328 & 1.43 & $1.4 \mathrm{e} 4$ & 1.2 & 0.6 \\
\hline 1996 & 227 & 1804 & 4.21 & $4.5 \mathrm{e} 3$ & 2.0 & 1.0 \\
\hline 1996 & 232 & 0953 & 4.23 & $3.0 \mathrm{e} 3$ & 1.6 & 0.8 \\
\hline 1997 & 317 & 2155 & 5.33 & $1.6 \mathrm{e} 3$ & 1.5 & 0.7 \\
\hline 1997 & 323 & 1645 & 5.34 & $1.1 \mathrm{e} 3$ & 1.3 & 0.6 \\
\hline 1998 & 128 & 2316 & 5.41 & $1.1 \mathrm{e} 3$ & 1.3 & 0.6 \\
\hline 1998 & 229 & 0739 & 5.36 & $2.0 \mathrm{e} 3$ & 1.7 & 0.8 \\
\hline 1998 & 236 & 0058 & 5.36 & $1.1 \mathrm{e} 3$ & 1.2 & 0.6 \\
\hline 1998 & 250 & 1607 & 5.35 & $1.2 \mathrm{e} 3$ & 1.3 & 0.6 \\
\hline 1998 & 291 & 2146 & 5.31 & $9.3 \mathrm{e} 2$ & 1.1 & 0.6 \\
\hline 1998 & 318 & 0543 & 5.27 & $2.1 \mathrm{e} 4$ & 5.6 & 3.1 \\
\hline
\end{tabular}


Table 2 (Continued)

\begin{tabular}{|c|c|c|c|c|c|c|}
\hline Year & Day & $\begin{array}{l}\text { GRB } \\
\text { time }\end{array}$ & $\begin{array}{l}\text { Ulysses } \\
R \text { (AU) }\end{array}$ & $\begin{array}{l}\text { Peak GRB } \\
\text { counts s }^{-1}\end{array}$ & $\begin{array}{l}\text { Inferred } \\
\text { X-class }\end{array}$ & $\begin{array}{l}+/- \\
\text { Error }\end{array}$ \\
\hline 1998 & 322 & 1250 & 5.27 & $2.6 \mathrm{e} 3$ & 1.9 & 1.0 \\
\hline 1998 & 330 & 0225 & 5.26 & $1.5 \mathrm{e} 3$ & 1.4 & 0.7 \\
\hline 1999 & 229 & 0519 & 4.66 & $1.3 \mathrm{e} 3$ & 1.2 & 0.6 \\
\hline 1999 & 230 & 0946 & 4.66 & $1.4 \mathrm{e} 3$ & 1.2 & 0.6 \\
\hline 1999 & 253 & 0755 & 4.59 & $3.3 \mathrm{e} 3$ & 1.9 & 1.0 \\
\hline 2000 & 132 & 1200 & 3.53 & $2.4 \mathrm{e} 3$ & 1.2 & 0.6 \\
\hline 2000 & 133 & 2338 & 3.53 & $6.7 \mathrm{e} 3$ & 2.1 & 1.0 \\
\hline 2000 & 211 & 1802 & 3.08 & $6.2 \mathrm{e} 3$ & 1.7 & 0.9 \\
\hline 2000 & 212 & 0347 & 3.07 & $3.3 \mathrm{e} 3$ & 1.2 & 0.6 \\
\hline 2000 & 212 & 1629 & 3.07 & $6.8 \mathrm{e} 3$ & 1.8 & 0.9 \\
\hline 2000 & 232 & 2142 & 2.95 & $4.6 \mathrm{e} 3$ & 1.4 & 0.7 \\
\hline 2000 & 256 & 1717 & 2.79 & $4.7 \mathrm{e} 3$ & 1.3 & 0.7 \\
\hline 2000 & 363 & 1151 & 2.06 & $3.9 \mathrm{e} 4$ & 2.9 & 1.5 \\
\hline 2001 & 108 & 0219 & 1.40 & $8.1 \mathrm{e} 5$ & 9.4 & 5.4 \\
\hline 2001 & 130 & 0043 & 1.35 & $3.6 \mathrm{e} 5$ & 5.9 & 3.3 \\
\hline 2001 & 170 & 0340 & 1.37 & $1.6 \mathrm{e} 4$ & 1.2 & 0.6 \\
\hline 2001 & 171 & 0344 & 1.38 & $3.1 \mathrm{e} 4$ & 1.7 & 0.9 \\
\hline 2001 & 171 & 1930 & 1.38 & $1.9 \mathrm{e} 4$ & 1.3 & 0.7 \\
\hline 2001 & 261 & 0151 & 1.85 & $1.4 \mathrm{e} 4$ & 1.5 & 0.8 \\
\hline 2002 & 99 & 0609 & 3.21 & $3.3 \mathrm{e} 3$ & 1.3 & 0.6 \\
\hline 2002 & 134 & 1434 & 3.41 & $2.2 \mathrm{e} 3$ & 1.1 & 0.6 \\
\hline 2002 & 197 & 1615 & 3.74 & $4.8 \mathrm{e} 3$ & 1.8 & 0.9 \\
\hline 2002 & 199 & 1938 & 3.75 & $4.5 \mathrm{e} 4$ & 5.9 & 3.3 \\
\hline 2002 & 200 & 1641 & 3.76 & $2.2 \mathrm{e} 3$ & 1.2 & 0.6 \\
\hline 2002 & 223 & 0432 & 3.87 & $5.8 \mathrm{e} 3$ & 2.1 & 1.1 \\
\hline 2002 & 223 & 0642 & 3.87 & $2.8 \mathrm{e} 3$ & 1.4 & 0.7 \\
\hline 2002 & 223 & 1424 & 3.87 & $4.3 \mathrm{e} 3$ & 1.8 & 0.9 \\
\hline 2002 & 239 & 1307 & 3.95 & $1.6 \mathrm{e} 3$ & 1.1 & 0.5 \\
\hline 2002 & 240 & 1834 & 3.95 & $3.1 \mathrm{e} 3$ & 1.5 & 0.8 \\
\hline 2002 & 284 & 0138 & 4.15 & $4.2 \mathrm{e} 3$ & 1.9 & 1.0 \\
\hline 2002 & 287 & 1451 & 4.16 & $4.3 \mathrm{e} 3$ & 1.9 & 1.0 \\
\hline 2002 & 291 & 0755 & 4.18 & $4.8 \mathrm{e} 3$ & 2.1 & 1.1 \\
\hline 2002 & 298 & 1004 & 4.20 & $3.0 \mathrm{e} 3$ & 1.6 & 0.8 \\
\hline 2003 & 161 & 0622 & 4.95 & $2.4 \mathrm{e} 3$ & 1.7 & 0.9 \\
\hline 2003 & 263 & 2238 & 5.16 & $1.4 \mathrm{e} 3$ & 1.4 & 0.7 \\
\hline 2003 & 264 & 0841 & 5.16 & $1.6 \mathrm{e} 3$ & 1.5 & 0.7 \\
\hline 2003 & 264 & 2102 & 5.16 & $2.8 \mathrm{e} 3$ & 1.9 & 1.0 \\
\hline 2003 & 280 & 2254 & 5.19 & $1.6 \mathrm{e} 3$ & 1.5 & 0.7 \\
\hline 2003 & 281 & 1853 & 5.19 & $3.4 \mathrm{e} 3$ & 2.2 & 1.1 \\
\hline 2003 & 291 & 1544 & 5.21 & $2.7 \mathrm{e} 3$ & 1.9 & 1.0 \\
\hline 2003 & 306 & 0946 & 5.23 & $2.1 \mathrm{e} 3$ & 1.7 & 0.8 \\
\hline 2003 & 310 & 1657 & 5.23 & $1.1 \mathrm{e} 3$ & 1.2 & 0.6 \\
\hline 2003 & 320 & 0607 & 5.25 & $1.3 \mathrm{e} 3$ & 1.3 & 0.6 \\
\hline
\end{tabular}




\section{Appendix A: Ulysses GRB Comparison with RHESSI}

The Ulysses observing time range overlapped with that of RHESSI, which gives us an opportunity for direct photometric comparisons of specific events (e.g., Kane, McTiernan, and Hurley, 2005). We have done a comparison for one well-observed flare, the GOES X8.3 of 2 November 2003. By comparing the corrected counting rates, we confirm a certain level of understanding of these corrections: thermal feed-through, gain (energy scale), pulse pile-up, and dead time, in particular.

Broad-band hard X-ray photometry of solar flares poses particular problems in reconciling the intense thermal component, usually characterized by a maximum $k T$ in the range $1-2 \mathrm{keV}$, and the much fainter non-thermal tail produced mainly by $10-100 \mathrm{keV}$ electrons. RHESSI partially solves this problem by having a set of shutters (Lin et al., 2002) that control the absorber thickness across the detector. All other solar hard X-ray spectrometers have fixed windows (that of Ulysses is $0.3 \mathrm{~mm} \mathrm{Al}$ in a hemispherical dome configuration). The entrance window serves as a high-pass filter which determines the distribution of pulse heights; in the case of GRB the half-transmission energy thus works out to be about $15 \mathrm{keV}$, but the steepness of the thermal source spectrum pushes the peak in the pulse-height spectrum to below $10 \mathrm{keV}$ for the lower temperatures. These counts can still contribute to the $25-150 \mathrm{keV}$ observational window, however, via resolution broadening and via pulse pileup (see Appendix B).

Figure 7 shows the essential elements for a model representation of the pulse-height spectrum produced by a solar thermal source. Note that the peak of the pulse-height distributions fall well below the electronics threshold, and even below the 50\% transmission energy of the entrance window. The high-energy tail of these pulse distributions competes with the true hard X-ray spectrum. This causes the morphologically smoother light curves seen at lower hard X-ray energies, seen not just with Ulysses GRB but with most other scintillator-based spectrometers.

The comparison of counting rates with RHESSI provides essentially complete diagnostics, since RHESSI has high spectral resolution and is not limited to a single telemetry band $(25-150 \mathrm{keV})$ as GRB is. We show the direct comparison in Figure 8, which illustrates a satisfactory comparison early in the event, then a growing divergence later on. The RHESSI live time reached a minimum of about $15 \%$ near the time of peak counting rates, and at $25 \mathrm{keV}$ also exhibited the smooth late excess - though not so large-as found in the GRB counts. This behavior can be explained by the poorer spectral resolution of GRB, which is determined by its on-board calibration to be about $48 \%$ at $25 \mathrm{keV}$. There is no need to invoke pulse pile-up for this event, and the dead time corrections for both instruments also seem satisfactory.

\section{Appendix B: The Effects of Pulse Pile-Up on the Ulysses GRB Detector}

Pulse pile-up is an effect that occurs at high count rates. Two or more pulses interact in the detector within a time that is too short for the electronics to distinguish them (Kane and Hudson, 1970; Datlowe, 1975). The resulting signal is a single pulse whose equivalent energy is roughly the sum of the energies of the input pulses. Even if the energies of the input pulses are below the electronics threshold for detection (see Figure 7), the resulting sum signal may be above it. This effect can be an important one when the input spectrum is from a solar flare because of the large fluxes of low-energy photons in the thermal component of the spectrum. These pulses are below the $25 \mathrm{keV}$ GRB counting threshold and so are 


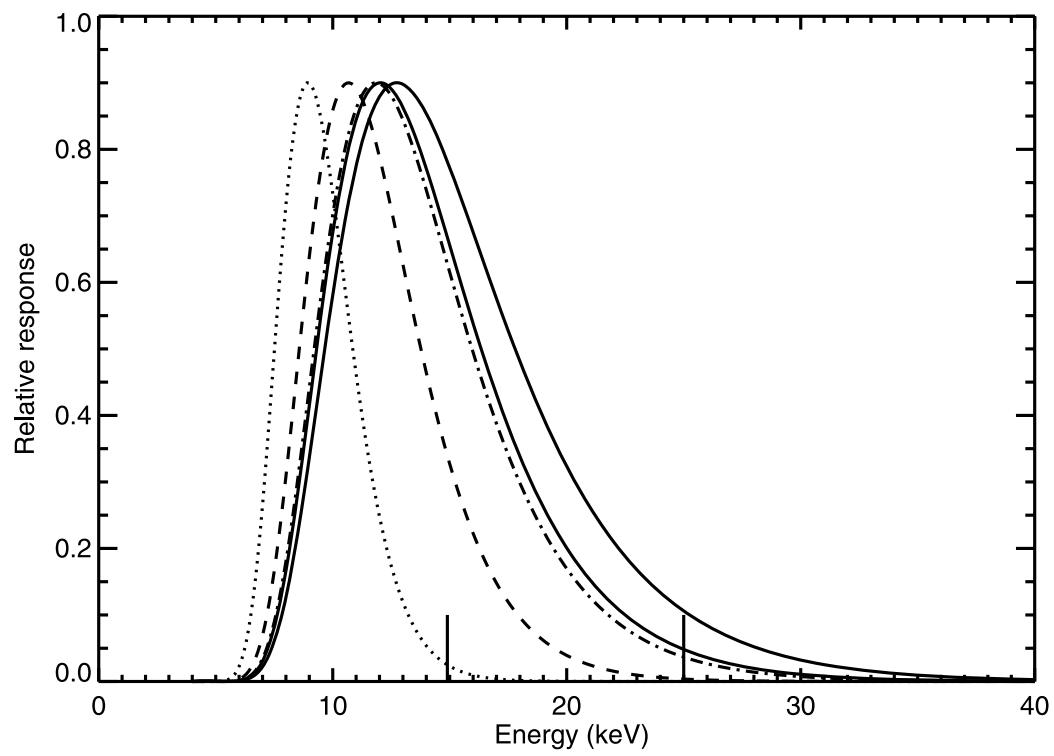

Figure 7 Model pulse height distributions for the Ulysses GRB detector for exponential input spectra with $k T=1,2,3,3.2$, and 4. The solid line $(k T=3.2)$ shows the spectrum chosen for the pile-up studies (Appendix B). The short vertical lines show (left) the minimum 50\% transmission energy for GRB, and (right) the electronic channel threshold of $25 \mathrm{keV}$.

not counted directly, but if multiple events occur within the detection time window, their sum pulse can exceed the threshold and be counted. The situation is further complicated by several factors. First, without knowledge of the shape and intensity of the thermal solar $\mathrm{X}$-ray spectrum, a correction cannot be calculated. Second, because the thermal counts are not directly detected, the shape of the input photon spectrum cannot be inferred from the observed pulse-height spectrum. Note that pulse pile-up is distinct from dead time effects, since the pulses that pile up do so within a dead time interval. Both effects can be present at high enough count rates. Also note that thermal feed-through, described above, depends only on the spectral distribution and should not be confused with rate-dependent effects.

Pile-up can be studied by Monte Carlo simulations. A very thorough Monte Carlo program was written for the Ulysses detector, and was used to infer the influence of pulse pile-up on the spectra of major flares (Kane et al., 1995). We have now used this program to estimate the effects of pile-up using RHESSI observations for the input spectrum, specifically those described by Holman et al. (2003). The thermal component of this flare had an effective temperature of $3.2 \mathrm{keV}$ at maximum. We have simulated the effect that such a spectrum would have on the GRB detector for input counting rates over a wide range, as described below.

The Monte Carlo program simulates the effects of a parallel beam of X-rays incident on the GRB detector on-axis (as a solar flux would approximately be). Compton and photoelectric K- and L-shell interactions in the hemispherical geometry of the CsI crystals are taken into account, as are Auger electrons, fluorescence, photon scattering out of the crystal, and the escape of scattered electrons. The crystal resolution as a function of energy, and the $0.03 \mathrm{~cm}$ thick Al window are also taken into account. For the simulations described here, a bremsstrahlung-like exponential photon spectrum was used as input between 1 and $150 \mathrm{keV}$. We refer to the input as photons, to distinguish it from the detected counts. The input photon 

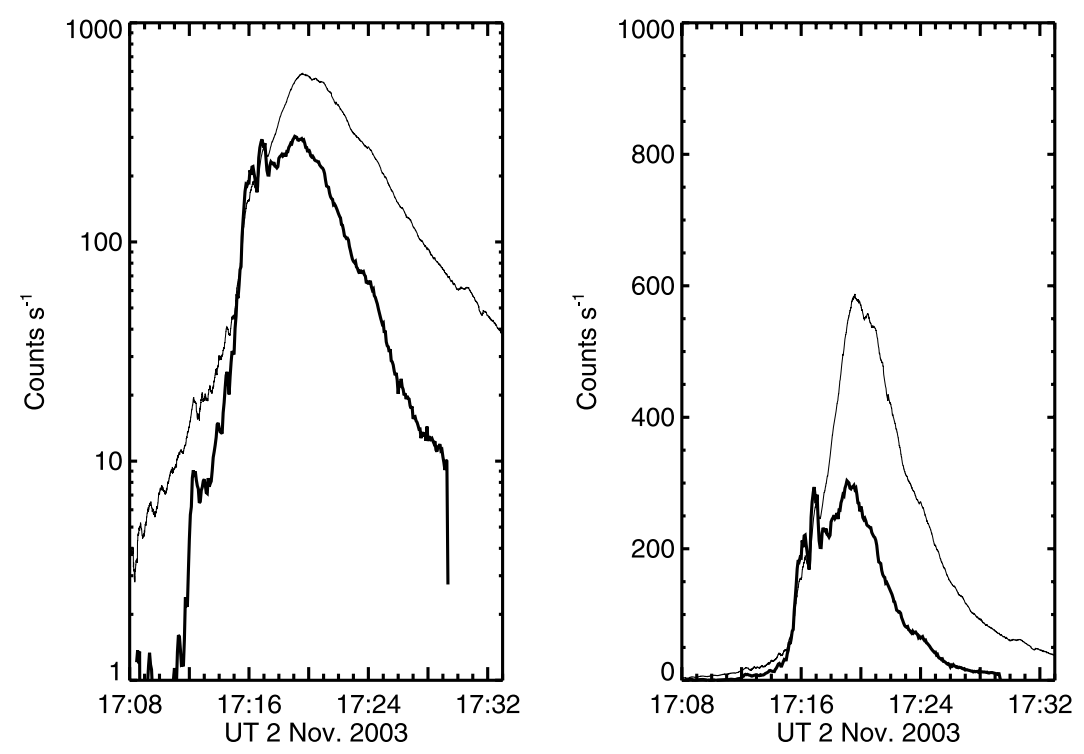

Figure 8 Comparison of RHESSI counting rate (thick line) with GRB counting rate (thin line and scaled by an arbitrary factor) for $25-150 \mathrm{keV}$ during the X8.3 flare of 2 November 2003: Logarithmic (left) and linear (right) plots. We attribute the discrepancy after about 17:17 UT to the increasing emission measure of the thermal component directly detected by GRB, rather than via pile-up. During the impulsive phase there is good agreement.

rate was varied between $10^{3}$ and $10^{7}$ photons $\mathrm{s}^{-1}$ with a Poisson interval distribution, and the detected count rate due to pile-up was recorded in the $25-150 \mathrm{keV}$ energy range. If two or three photons interacted in the detector within a time interval less than one microsecond (the approximate shaping time constant for the GRB detector), they were counted as a single, piled-up count whose energy was the sum of the energies left by the original photons. More than three pile-ups were not tracked, but they will be an important component at very high input count rates. Each simulation used $10^{6}$ input photons.

Figure 9 shows the input photon and output count spectra for inputs of $10^{3}$ and $10^{7}$ photons $\mathrm{s}^{-1}$. At $10^{3}$ photons $\mathrm{s}^{-1}$, the output count spectrum follows the input photon spectrum above an energy of about $20 \mathrm{keV}$. Below this energy, the input photon spectrum is strongly attenuated due to the $\mathrm{Al}$ entrance window (for a parallel input beam, its effective thickness is $0.03 \mathrm{~cm}$ only for a single, on-axis point, and increases off-axis due to the hemispherical geometry). At $10^{7}$ photons $\mathrm{s}^{-1}$, the output count spectrum is attenuated severely below about $35 \mathrm{keV}$, due to photon interactions which pile up and are counted at higher energies. The count spectrum above $35 \mathrm{keV}$ clearly reflects this phenomenon.

Figure 10 summarizes some of these results. The bottom $x$-axis shows the input photon rate at the GRB detector over the $1-150 \mathrm{keV}$ range for a bremsstrahlung-type exponential spectrum with $k T=3.2 \mathrm{keV}$. This is converted into $\mathrm{W} \mathrm{m}^{-2}$ at $1 \mathrm{AU}$ in the GOES soft channel along (the upper $x$-axis), so that the count rates can be compared to GOES fluxes, using the scaling law derived in Section 4.2. The $y$-axis shows the piled-up count rate for the GRB experiment in the $25-150 \mathrm{keV}$ range. The piled-up count rate is calculated for assumed GRB solar distances of $1 \mathrm{AU}$ and 3.2 AU in Figure 10. The figures show that pile-up is negligible

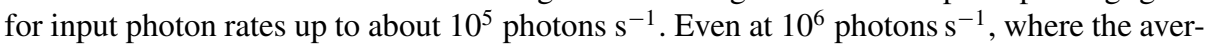
age interval between photons is only $1 \mu \mathrm{s}$, that is, one pulse-shaping time, the piled-up count 

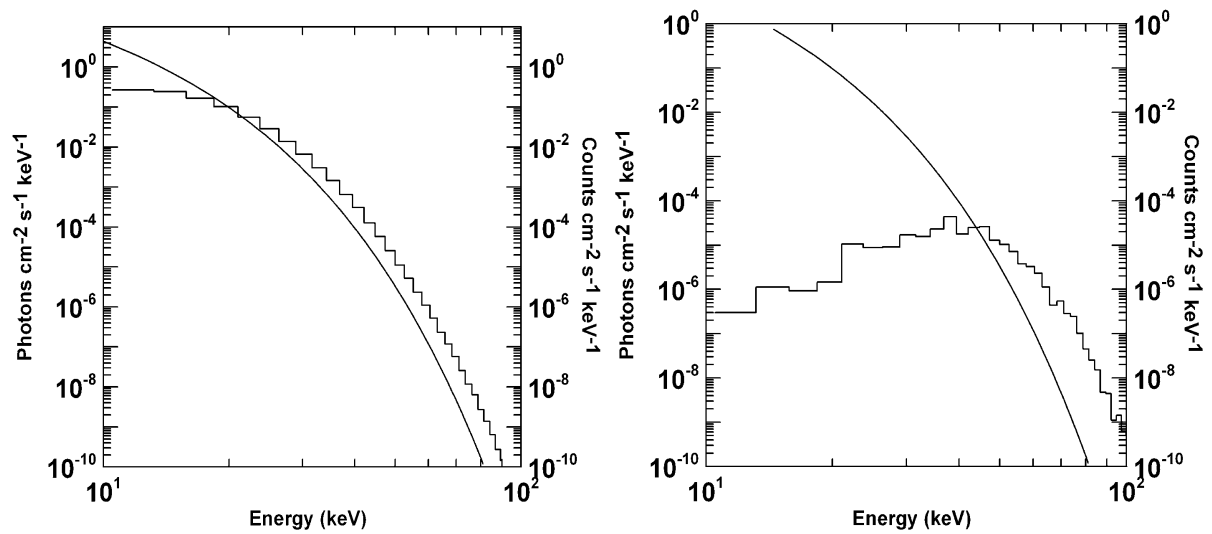

Figure 9 Smooth curve (left hand axis): a $3.2 \mathrm{keV}$ bremsstrahlung photon spectrum, used as the input to the detector. Histogram (right hand axis): the resulting count spectra in the GRB detector, calculated by the Monte Carlo program. Input rates of $10^{3}$ photons s $^{-1}$ (left hand figure) and $10^{7}$ photons s ${ }^{-1}$ (right hand

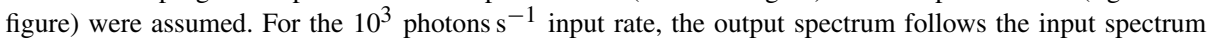
above about $20 \mathrm{keV}$, and is attenuated below it due to the entrance window. Pile-up is negligible. For the higher input photon rate, the output spectrum is severely attenuated below about $35 \mathrm{keV}$ due to pile-up, and many of these piled-up photons appear between 35 and $200 \mathrm{keV}$. The count spectrum lies above the photon spectrum because of the poor energy resolution of the GRB detector resulting from its geometry. As a consequence, there is a tendency for the detector to dump more low energy counts into the higher energy bins, than high energy counts into the lower energy bins.
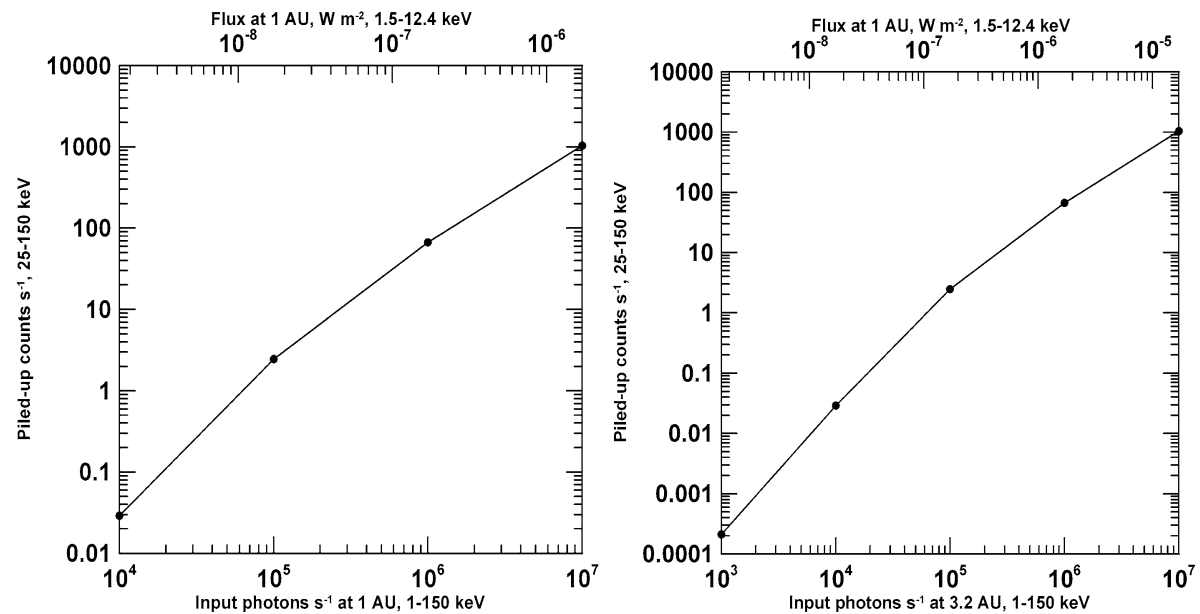

Figure 10 Summary of the Monte Carlo results for Ulysses at $1 \mathrm{AU}$ (left) and 3.2 AU (right) from the Sun. A $3.2 \mathrm{keV}$ thermal bremsstrahlung spectrum was assumed, and one million photons were tracked. The lower $x$-axis gives the input photon rate over the GRB detector between 1 and $150 \mathrm{keV}$. The $y$-axis shows the rate of double and triple pile-ups in the $25-150 \mathrm{keV}$ GRB energy band. The top $x$-axis gives the equivalent flux for a $3.2 \mathrm{keV}$ bremsstrahlung spectrum in the $1.5-12.4 \mathrm{keV}$ band, for comparison with GOES rates.

rate is small compared to the input photon rate. This can be understood as the result of several factors. First, the input photon rate is measured over a wider energy range, $1-150 \mathrm{keV}$, than the piled-up count rate $(25-150 \mathrm{keV}$, the GRB passband). Second, for photons to ac- 
tually pile up into the GRB passband, they must traverse the entrance window and deposit energies whose sum falls between 25 and $150 \mathrm{keV}$; many interactions, although piled-up, result in smaller energy deposits, and would not be counted. Finally, the input photon fluxes are quite small at energies above $20 \mathrm{keV}$ compared to the $1 \mathrm{keV}$ fluxes, due to the shape of the bremsstrahlung spectrum; thus, while they can pile up and fall into the passband, their numbers are very small. We emphasize that two effects have not been treated here. The first is the pile-up of more than three simultaneous pulses. This will become important at input photon rates above $\approx 3 \times 10^{5}$ counts $^{-1}$, and therefore the piled-up count rates in Figure 10 at these input photon rates should be considered very conservative lower limits. The second is the pile-up of thermal input photons with non-thermal ones; we have not assumed any non-thermal component in these simulations.

\section{References}

Battaglia, M., Grigis, P.C., Benz, A.O.: 2005, Astron. Astrophys. 439, 737.

Brodrick, D., Tingay, S., Wieringa, M.: 2005, J. Geophys. Res. (Space Phys.) 110, 9.

Cliver, E.W., Dennis, B.R., Kiplinger, A.L., Kane, S.R., Neidig, D.F., Sheeley, N.R. Jr., Koomen, M.J.: 1986, Astrophys. J. 305, 920.

Crawford, D.F., Jauncey, D.L., Murdoch, H.S.: 1970, Astrophys. J. 162, 405.

Crosby, N., Aschwanden, M. Dennis, B.R.: 1993, Solar Phys. 143, 275.

Crosby, N., Vilmer, N., Lund, N., Sunyaev, R.: 1998, Astron. Astrophys. 334, 299.

Datlowe, D.W.: 1975, Space Sci. Inst. 1, 389.

Dennis, B.R.: 1985, Solar Phys. 100, 465.

Dennis, B.R.: 1988, Solar Phys. 118, 49.

Dennis, B.R., Zarro, D.M.: 1993, Solar Phys. 146, 177.

Feldman, U.: 1996, Phys. Plasmas, 3, 3203.

Frost, K.J., Dennis, B.R.: 1971, Astrophys. J. 165, 655.

Garcia, H.A.: 1990, Solar Phys. 127, 185.

Garcia, H.A., McIntosh, P.S.: 1992, Solar Phys. 141, 109.

Holman, G.D., Sui, L., Sui, L., Schwartz, R.A., Emslie, A.G.: 2003, Astrophys. J. 595, L97.

Hudson, H.S.: 1978, Astrophys. J. 224, 235.

Hudson, H.S.: 1991, Solar Phys. 133, 357.

Hudson, H., Ryan, J.: 1995, Annu. Rev. Astron. Astrophys. 33, 239.

Hurley K.: 1986, In: Marsden, R.G. (ed.) The Sun and the Heliosphere in Three Dimensions, Reidel, Dordrecht, 73.

Hurley, K., Sommer, M., Atteia, J.L., Boer, M., Cline, T., Cotin, F., Henoux, J.C., Kane, S., Lowes, P., Niel, M.: 1992, Astron. Astrophys. 92, 401.

Kane, S.R., Hudson, H.S.: 1970, Solar Phys. 14, 414.

Kane, S.R., McTiernan, J.M., Hurley, K.: 2005, Astron. Astrophys. 433, 1133.

Kane S.R., Crannell C.J., Datlowe D., Feldman U., Gabriel A., Hudson H.S., Kundu M.R., Maetzler C., Neidig D., Petrosian V.: 1980, In: Sturrock, P.A. (ed.) Solar Flares: A Monograph from Skylab Solar Workshop II., Colorado Assoc. Univ. Press, Boulder, 187.

Kane, S.R., Hurley, K., McTiernan, J.M., Sommer, M., Boer, M., Niel, M.: 1995, Astrophys. J. 446, L47.

Kane, S.R., Hurley, K., McTiernan, J.M., Boer, M., Niel, M., Kosugi, T., Yoshimori, M.: 1998, Astrophys. J. 500, 1003.

Kiplinger A.L., Garcia H.A.: 2004, Bull. Am. Astron. Soc. 36, 739.

Kucera, T.A., Dennis, B.R., Schwartz, R.A., Shaw, D.: 1997, Astrophys. J. 475, 338.

Lin, R.P., Schwartz, R.A., Pelling, R.M., Hurley, K.C.: 1981, Astrophys. J. 251, L109.

Lin, R.P., Dennis, B.R., Hurford, G.J., Smith, D.M., Zehnder, A., Harvey, P.R., et al.: 2002, Solar Phys. $210,3$.

Lingenfelter R.E., Hudson H.S.: 1980, In: Pepin, R.O., Eddy, J.A., Merrill, R.B. (eds.) The Ancient Sun: Fossil Record in the Earth, Moon and Meteorites, Pergamon Press, Elmsford, 69.

Nakajima, H., Dennis, B.R., Hoyng, P., Nelson, G., Kosugi, T., Kai, K.: 1985, Astrophys. J. 288, 806.

Neupert, W.M.: 1968, Astrophys. J. 153, L59.

Orwig, L.E., Frost, K.J., Dennis, B.R.: 1980, Solar Phys. 65, 25.

Osten, R.A., Drake, S., Tueller, J., Cummings, J., Perri, M., Moretti, A., Covino, S.: 2007, Astrophys. J. 654, 1052. 
Reedy R.C.: 1996, In: Balasubramaniam, K.S., Keil, S.L., Smartt, R.N. (eds.) Solar Drivers of the Interplanetary and Terrestrial Disturbances CS-95, Astron. Soc. Pac., San Francisco, 429.

Reedy R.C., Marti K.: 1991, In: Sonett, C.P., Giampapa, M.S., Matthews, M.S. (eds.) The Sun in Time, Univ. Arizona Press, Tucson, 260.

Smith H.J., Smith E.V.P.: 1963, Solar Flares, Macmillan, New York.

Thomson, N.R., Rodger, C.J., Dowden, R.L.: 2004, Geophys. Res. Lett. 31, L06803.

Tomczak, M.: 2001, Astron. Astrophys. 366, 294.

Veronig, A., Temmer, M., Hanslmeier, A., Otruba, W., Messerotti, M.: 2002, Astron. Astrophys. 382, 1070. 